\title{
Implementation and Evaluation of an Embedded LES-RANS Solver
}

\author{
Kameswararao Anupindi . \\ Richard D. Sandberg
}

Received: date / Accepted: date

\begin{abstract}
In the current work, we present the development and application of an embedded large-eddy simulation (LES) - Reynolds-averaged Navier Stokes (RANS) solver. The novelty of the present work lies in fully embedding the LES region inside a global RANS region through an explicit coupling at the arbitrary mesh interfaces, exchanging flow and turbulence quantities. In particular, a digital filter method (DFM) extracting mean flow, turbulent kinetic energy and Reynolds stress profiles from the RANS region is used to provide meaningful turbulent fluctuations to the LES region. The framework is developed in the open-source computational fluid dynamics software OpenFOAM. The embedding approach is developed and validated by simulating a spatially developing turbulent channel flow. Thereafter, flow over a surface mounted spanwise-periodic vertical fence is simulated to demonstrate the importance of the DFM and the effect of the location of the RANS-LES interface. Mean and second-order statistics are compared with direct numerical simulation (DNS) data from the literature. Results indicate that feeding synthetic turbulence at the LES interface is essential to achieve good agreement for the mean flow quantities. However, in order to obtain a good match for the Reynolds stresses, the LES interface needs to be placed sufficiently far upstream, which in the present case was six spoiler heights before the fence. Further, a realistic spoiler configuration with finite-width in the spanwise direction and inclined at 30 degrees was simulated using the embedding approach. As opposed to the vertical fence case this is a genuinely (statistically) three-dimensional case and a very good match with mean and second-order statistics was obtained with the experimental data. Finally, in order to test the present solver for high subsonic speed flows the flow over an open cavity was simulated. A good match with reference data is obtained for mean and turbulence profile comparisons. Tones in
\end{abstract}

K. Anupindi

Faculty of Engineering and the Environment, University of Southampton, SO17 1BJ, UK

Present address: Department of Mechanical Engineering, Indian Institute of Technology Madras, Chennai, 600036, India

E-mail: kanupindi@iitm.ac.in

R. D. Sandberg

Department of Mechanical Engineering, University of Melbourne, 3010, Victoria, Australia E-mail: richard.sandberg@unimelb.edu.au 
the pressure spectra were predicted reasonably well and an overall sound pressure level with a maximum deviation of $2.6 \mathrm{~dB}$ was obtained with the present solver when compared with the experimental data.

Keywords Embedded large-eddy simulation - Synthetic turbulence generation . Channel flow $\cdot$ Vertical fence $\cdot$ Inclined spoiler · Open cavity

\section{Introduction}

Reynolds-averaged Navier-Stokes (RANS) based simulations are a preferred choice in industry for simulating turbulent flows owing to their quick turn-over times. However, as the entire turbulence energy spectrum is modelled with underlying fully developed turbulent flow assumptions, it is difficult, at least for some simple models, to predict flows that involve complex geometrical and physical flow features such as sharp edges, flow-control devices, separation and re-attachment. Large-eddy simulations (LES) offer an alternative to this by resolving most of the turbulence energy spectrum and by only modelling the small-scale eddies in the dissipation range [28]. The effect of these small-scale unresolved eddies on the resolved scales is modelled through a sub-grid scale (SGS) model that closes the filtered set of Navier-Stokes equations. As only a small portion of the turbulence energy spectrum is modelled, LES provides better results even in complex flow cases, however, at the cost of higher grid resolution and therefore computational cost requirements when compared with RANS models. In order to strike a balance between the amount of modelling and the computational time required, several efforts in the past have considered modelling the near-wall region in RANS mode and separated flow regions in LES mode, as for example in detached eddy simulations (DES) [42]. Several variants and improvements of DES exist in the literature [43, 2]. By taking advantage of the structural similarity of the RANS and LES equations, other unified LES/RANS or blended LES-RANS methods have also been developed. In these methods the flow dynamically switches between RANS and LES based on the grid-resolution and other criteria defined in the blending functions $[1,3,46]$. These hybrid methods almost exclusively couple a RANS near-wall layer to an LES domain above it, in most cases dramatically improving accuracy relative to pure RANS and poorly resolved LES when applied to separated flows. However, in the context of flow control, involving complex flow regions around actuators, probes or other protuberances, the opposite might be required, namely the embedding of LES zones within the wall region to resolve important flow features close to the wall.

Thus the present work is focused on another hybrid RANS/LES philosophy, known as zonal RANS - LES methods in which the interface between the RANS and the LES is defined a priori before the simulation starts and it remains fixed for the entire simulation $[29,12]$. The respective statistically averaged equations are solved in the RANS region and filtered equations are solved in the LES region. Coupling at the interface between the regions poses additional challenges in terms of transferring the flow and turbulence quantities both ways. As the embedding approach can be based on coupling two separate solvers, several advantages emerge, such as selecting individual spatial and temporal discretisation schemes and that different dimensionality for each of the flow solvers can be used as was done by 
Quéméré and Sagaut [29]. In the following, we discuss some previous studies in the category of the zonal LES-RANS method followed by goals and novel aspects of the present study. The numerical methodology is described in section 2 , the results are presented in section 3 , and conclusions and outlook are reported in section 4.

\subsection{Background}

\subsubsection{Previous studies}

There have been several past studies that focused on zonal RANS-LES solvers. Quéméré and Sagaut [29] presented zonal multi-domain RANS/LES simulations for plane channel flow and a plate with finite thickness and a blunt trailing-edge. In another study, König et al. [12] evaluated three different methods to couple the interface between RANS and LES regions. However, their simulations were limited to Spalart-Allmaras one-equation turbulence model in the RANS region and monotone integrated large-eddy simulation (MILES) in the LES region. Further their solver was extended to study supersonic flat plate boundary layer and shock-wave turbulent boundary-layer interaction by Roidl et al. [32]. Zhang et al. [50] applied the zonal RANS-LES method to compute the flow over an airfoil in high-lift configuration. In their method, a two-dimensional RANS solution is computed first for the full region (including the LES region) and the necessary boundary conditions for the embedded LES domain are extracted. Such a method can be efficiently applied to spanwise periodic problems only, for problems involving geometrical three-dimensionality the precursor RANS simulation also needs to be three-dimensional which increases the cost of the computation. Xiao and Jenny [48] developed a consistent dual-mesh framework for hybrid LES - RANS modelling in OpenFOAM. The need to generate two different meshes meeting the demands of individual RANS and LES regions for the entire flow geometry might pose constraints in extending such a dual-mesh method to simulate flows in complex geometries. Recently, Deck [2] presented a zonal detached eddy simulation (ZDES) framework. This framework generalises the detached eddy simulation (DES) in a way by allowing to select RANS and DES regions a priori and a hybrid length scale that goes with the DES depending on the problem of interest. Mathey [20] applied a zonal RANS-LES approach for the prediction of broadband and tonal noise generated by the flow past an airfoil trailing edge. In their formulation, they directly injected the RANS values to update the LES values on the interface boundaries and vice versa. It was however, not specified how the turbulence transport variables are updated on the RANS side of the interface. Richez et al. [30] performed zonal RANS/LES coupling simulation for transitional and separated flow around an airfoil near stall. They used overlapping RANS and LES zones to cover the computational domain. They achieved a good agreement for the calculated aerodynamic characteristics when compared with the experimental data. Roidl et al. [33] presented a reformulated synthetic fluctuation generation and applied it to study zero pressure gradient boundary layers. Subsequently, they tested the method for favourable and adverse pressure gradients as well [34]. The list of previous studies covered here is certainly not complete, hence readers are referred to the review article by Fröhlich and von Terzi [4] and the book by Sagaut et 
al. [38]. A survey of the literature suggests that there is a need to develop and validate an embedded LES - RANS framework that can simulate complex industrial flow problems.

\subsubsection{Goals and novel aspects of the present work}

The goal of the present work is to develop a framework to embed scale-resolving LES regions inside global RANS regions, in particular to allow for LES regions at the wall. The embedding of eddy-resolving LES regions inside RANS regions can be seen as the opposite of using a RANS model in the near-wall region for high Reynolds number flows. The novelty of the present work is to fully embed the LES regions inside RANS regions without using any overlap regions between them and to feed meaningful small scale fluctuations at the LES interface in a fully coupled manner by extracting mean and Reynolds stress profiles from the upstream RANS region that can adapt to variations in time. Further, another aim of the present work is to be able to generate the RANS and LES meshes with required resolutions and couple them through non-conformal interfaces. This latter goal is hoped to ease mesh generation for complex industrial application cases. Additional novel aspects that the manuscript offers are the following (i) Usage of no overlapping zones between the RANS and LES regions thus avoiding any grey regions (ii) A two-equation RANS model $(k-\omega$ SST) was coupled with a lineareddy viscosity type sub-grid-scale model (Smagorinsky) (iii) Three incompressible and one compressible case, all featuring different characteristics and using different metrics for assessing the simulation quality, were simulated and a good match with the reference data was obtained in each case. The solver is evaluated by subjecting it to different challenging environments through each of these test cases.

\section{Numerical Methodology}

\subsection{Governing Equations}

The governing equations are the unsteady, three-dimensional, compressible NavierStokes equations. Applying one of the scale separation operators, statistical averaging in the case of RANS or spatial filtering in the case of LES, the conservation of mass, momentum and total energy that are solved can be written as follows,

$$
\begin{gathered}
\frac{\partial \bar{\rho}}{\partial t}+\frac{\partial}{\partial x_{j}}\left(\bar{\rho} \widehat{u_{j}}\right)=0 \\
\frac{\partial\left(\bar{\rho} \widehat{u_{i}}\right)}{\partial t}+\frac{\partial}{\partial x_{j}}\left(\bar{\rho} \widehat{u_{i}} \widehat{u_{j}}\right)=-\frac{\partial \bar{p}}{\partial x_{i}}+\frac{\partial}{\partial x_{j}}\left(\mu\left(\frac{\partial \widehat{u_{i}}}{\partial x_{j}}+\frac{\partial \widehat{u_{j}}}{\partial x_{i}}\right)-\frac{2}{3} \mu \frac{\partial \widehat{u_{j}}}{\partial x_{j}} \delta_{i j}\right)+\frac{\partial \widehat{\tau_{i j}^{h y b}}}{\partial x_{j}}(2) \\
\frac{\partial(\bar{\rho} \widehat{E})}{\partial t}+\frac{\partial}{\partial x_{j}}\left(\bar{\rho} \widehat{E} \widehat{u_{j}}\right)=-\frac{\partial}{\partial x_{j}}\left(\bar{p} \widehat{u_{j}}\right)+\frac{\partial}{\partial x_{j}}\left[\widehat{u_{i}}\left(\mu\left(\frac{\partial \widehat{u_{i}}}{\partial x_{j}}+\frac{\partial \widehat{u_{j}}}{\partial x_{i}}\right)-\frac{2}{3} \mu \frac{\partial \widehat{u_{j}}}{\partial x_{j}} \delta_{i j}\right)\right] \\
+\frac{\partial}{\partial x_{j}}\left(\kappa \frac{\partial \widehat{T}}{\partial x_{j}}\right)+\frac{\partial \tau_{i j}^{h y b} \widehat{u_{i}}}{\partial x_{j}}+\widehat{\tau_{i j}^{h y b}} \frac{\partial \widehat{u_{i}}}{\partial x_{j}}-\frac{\partial q_{j}^{h y b}}{\partial x_{j}}
\end{gathered}
$$


where $u, p, \rho, T, E, \mu$ and $\kappa$ are fluid velocity, pressure, density, temperature, total energy, dynamic viscosity and thermal conductivity, respectively. The fluid is assumed to be an ideal gas with constant specific heat coefficients. The additional terms that arise in the discretised governing equations due to the non-linear terms are indicated with $h y b$ superscript. These are modelled using the respective turbulence models depending on the region. The hat and bar operators on the variables in the Eqs. 1-3 represent Favre-filtering in the case of LES, whereas they represent statistical averaging in the case of RANS.

\subsection{Turbulence Models}

In the present work the $k-\omega$ shear stress transport (SST) model is used as the turbulence model in the RANS region and the classical Smagorinsky model is used as the SGS model in the LES region. The details of each of the models used is presented in the following sub sections.

\subsection{1 $k-\omega$ Shear Stress Transport Model}

Menter's shear stress transport (SST) model [22] is a linear eddy viscosity model and the Reynolds stress tensor is represented as

$$
\overline{\tau_{i j}}=\frac{2}{3} \rho k \delta_{i j}-2 \mu_{t}\left(\overline{S_{i j}}-\frac{1}{3} \overline{S_{k k}} \delta_{i j}\right)
$$

where $\mu_{t}$ is the turbulent viscosity to be determined from the underlying RANS model used, $k=\frac{1}{2} \overline{u_{i}^{\prime \prime} u_{i}^{\prime \prime}}$ is the turbulent kinetic energy, $u_{i}^{\prime \prime}$ is the fluctuating velocity and the overbar operator denotes statistical averaging. Similarly the turbulent heat flux term, $\widehat{q_{j}^{h y b}}$, appearing in Eq. 3, is modelled as

$$
\overline{q_{j}}=-\frac{\mu_{t} C_{p}}{P r_{t}} \frac{\partial \bar{T}}{\partial x_{j}}
$$

where $\mathrm{Pr}_{t}$ and $C_{p}$ represent turbulent Prandtl number and specific heat at constant pressure respectively. The turbulent Prandtl number is taken as 0.7 in the present computations. In this model two additional transport equations, turbulent kinetic energy $(k)$ and turbulent frequency $(\omega)$ are solved in order to estimate the turbulent viscosity $\left(\mu_{t}\right)$. The two-equation model of Menter [22] is used for the same.

\subsubsection{Smagorinsky Sub-Grid Scale Model}

In the case of LES, the stress tensor resulting from the filtering operation $\widehat{\tau_{i j}^{h y b}}$ is represented as $\widetilde{\tau_{i j}}$, where tilde represents a Favre filtering process. Using the Boussinesq hypothesis the stress tensor is modelled as

$$
\widetilde{\tau_{i j}}-\frac{1}{3} \widetilde{\tau_{k k}} \delta_{i j}=-2 \mu_{S G S}\left(\widetilde{S_{i j}}-\frac{1}{3} \widetilde{S_{k k}} \delta_{i j}\right)
$$

where $\mu_{S G S}$ is the sub-grid eddy viscosity, the tilde operator denotes Favre averaging, $\widetilde{S_{i j}}$ is Favre filtered strain rate tensor and the iso-tropic part of the stress 
tensor $\tau_{k k}$ is absorbed into the static pressure term in Eq. 2. The sub-grid heat flux term, $\widehat{q_{j}^{h y b}}$, in Eq. 3 is modelled in a similar manner to the stress tensor as

$$
\widehat{q_{j}^{h y b}}=\widetilde{q_{j}}=-\frac{\mu_{S G S} C_{p}}{P r_{t}} \frac{\partial \widetilde{T}}{\partial x_{j}} .
$$

Now depending on how $\mu_{S G S}$ is evaluated several models can be defined. The simplest and the first model that was proposed in the literature is the Smagorinsky model [28]. In this model the sub-grid viscosity is modelled as

$$
\mu_{S G S}=\rho\left(C_{s} \Delta\right)^{2} \sqrt{2 \widetilde{S_{i j} \widetilde{S_{i j}}}}
$$

where $C_{s}$ is a constant for the Smagorinsky model that is set to 0.065 and $\Delta$ is the local mesh size taken to be cube root of the volume of the cell.

\subsection{Interface Treatment}

The interface between the RANS and LES regions is the crucial element in embeddedtype hybrid turbulence models. In the following, the two-way coupling procedure developed and used in the present work is discussed.

\subsubsection{Going from RANS to LES}

The LES region needs to be provided with both mean flow variables and turbulent fluctuations in order for the turbulence to sustain. As RANS modelling works in a deterministic way, the data from the RANS side of the interface consists of the time averaged flow quantities and the turbulence transport variables (in the present case $k$ and $\omega$ ) and the Reynolds stress tensor. The mean flow quantities obtained from the RANS region are linearly interpolated to the LES interface, however, we also need a mechanism to supply meaningful turbulence fluctuations at the LES inflow interface. There are several ways to generate or extract these fluctuations, such as (i) running an auxiliary precursor simulation, (ii) using a recycling technique in a part of the computational domain, or (iii) supplying synthetic turbulence as an addition to the mean velocity profiles. In the present work, we use the latter method. The generation of synthetic fluctuations is a challenging problem both for pure LES and embedded LES-RANS computations. An ideal method of generating synthetic fluctuations should produce as identical results as possible whether it is used as an inflow condition or as an interface condition. A variety of methods to generate synthetic turbulence exist in the literature [38] such as (a) spectral (b) synthetic eddy and (c) digital filter methods. As the focus of the present work is to embed eddy resolving methods inside RANS regions it is necessary to choose a method that is computationally inexpensive and provides meaningful, physical, turbulence fluctuations within a short distance from the place of introducing them into the flow. In the current work, we use the DFM variant developed by Xie and Castro [49]. The DFM was originally developed by Klein et al. [10]. In the variant developed by Xie and Castro [49], the auto-correlation function is assumed to have a form closer to exponential than Gaussian, as originally proposed by Klein et al. [10], and also the filtering of a full three-dimensional field is avoided. In Kim 
et al. [9] this variant was further extended such that the generated small-scale field is divergence free at every time step of the simulation. In the present work, as a compressible solver is employed the divergence free criterion is thought to be not particularly necessary and hence the version reported in Xie and Castro [49] is implemented. For the sake of completeness, the DFM is briefly described below and the readers are referred to the references $[10,49,9]$ for a full description:

- Generate normally distributed white-noise with zero-mean and unit-variance and choose integral length scales for each of the velocity components for each of the co-ordinate directions.

- By assuming an exponential form for the auto-correlation function, calculate the convolution filter coefficients from the chosen integral length scales and the grid spacing.

- Filter the white-noise using the computed coefficients to obtain $\phi_{i}(y, z, t)$ and at every time step, assuming $u_{j}^{*}(y, z, t=0)=0$, correlate this filtered field with the previous time level values to obtain the perturbation field:

$$
u_{j}^{*}(y, z, t+\Delta t)=u_{j}^{*}(y, z, t) e^{\left(\frac{-\pi U \Delta t}{4 I_{i 1}}\right)}+\phi_{i}(y, z, t) \sqrt{1-e^{\left(\frac{-\pi U \Delta t}{2 I_{i 1}}\right)}}
$$

where $I_{i 1}$ are the integral length scales in the streamwise $(x)$ direction, $U$ is the streamwise convection velocity, and $\Delta t$ is the time-step of the simulation.

- The perturbation field obtained above satisfies the two-point correlations through the prescribed integral length scales, now the amplitude of the perturbation field is scaled using an amplitude tensor and added to the linearly interpolated RANS mean velocity to obtain the velocities on the LES interface as follows:

$$
\widetilde{u}_{j}(y, z, t)^{L E S}=\mathcal{I}_{R L}\left[\bar{u}_{j}(y, z, t)^{R A N S}\right]+a_{i j}(y, z) u_{i}^{*}(y, z, t),
$$

where the amplitude tensor $a_{i j}(y, z)$ is computed from the Reynolds stresses $R_{i j}$, obtained from the upstream RANS region, proposed by Lund et al. [19] as follows:

$$
a_{i j}=\left(\begin{array}{ccc}
\sqrt{R_{11}} & 0 & 0 \\
R_{21} / a_{11} & \sqrt{R_{22}-a_{21}^{2}} & 0 \\
R_{31} / a_{11} & \left(R_{32}-a_{21} a_{31}\right) / a_{22} & \sqrt{R_{33}-a_{31}^{2}-a_{32}^{2}}
\end{array}\right)
$$

As the Mach number of the considered cases is sub-sonic, thermodynamic fluctuations are not introduced and the rest of the flow variables are linearly interpolated (represented by the interpolation operator $\mathcal{I}_{R L}$ ) to the fine-grid LES interface with the data from the coarse-grid RANS interface as

$$
\left[\begin{array}{ccc}
\widetilde{p} & \widetilde{\rho} & \widetilde{T}
\end{array}\right]^{L E S}=\mathcal{I}_{R L}\left[\begin{array}{lll}
\bar{p} & \bar{\rho} & \bar{T}
\end{array}\right]^{R A N S} .
$$

For a discussion on introducing thermodynamic fluctuations using the DFM see the article by Touber \& Sandham [44]. To the authors' knowledge, this approach has so far only been used to generate inflow turbulence. The novelty of the current work is to extract the mean flow, turbulence kinetic energy and Reynolds stresses in a time-dependent fashion from the upstream RANS region and use them in the synthetic turbulence generation in a fully coupled manner. Thus the synthetic turbulence generation depends on the upstream flow history and can adapt to variations in time. 
The flow that is entering the RANS region from the LES region is provided with boundary conditions for $k$ and $\omega$ as described in König et al. [12], as follows,

$$
\begin{aligned}
k^{\mathrm{RANS}} & =\mathcal{C}_{\mathrm{LR}}\left[k_{\text {resolved }}^{\text {LES }}\right]=\mathcal{C}_{\mathrm{LR}}\left[\frac{1}{2}\left\langle u_{i}^{\prime} u_{i}^{\prime}\right\rangle\right], \\
\omega^{\mathrm{RANS}} & =\mathcal{C}_{\mathrm{LR}}\left[\frac{1}{\sqrt{\beta_{c}}} \sqrt{2 \tilde{S_{i j}} \tilde{S_{i j}}}\right],
\end{aligned}
$$

where $u_{i}^{\prime}$ denotes the sub-grid scale velocity, the $\langle$.$\rangle operator denotes a time-average$ and $\beta_{c}=0.09$ is the same as in reference [12]. The calculation of turbulent kinetic energy on the LES side is straightforward whereas the quantity $\omega$ is approximated by a generalised form of Bradshaw's hypothesis [24,12], using the strain rate tensor $\tilde{S}_{i j}=\frac{1}{2}\left(\frac{\partial \tilde{u}_{i}}{\partial x_{j}}+\frac{\partial \tilde{u}_{j}}{\partial x_{i}}\right)$. All other flow quantities are linearly restricted (represented by the coarsening operator $\mathcal{C}_{L R}$ ) from the fine LES interface to the coarse RANS interface and are also time averaged by a moving average with a period $\Delta T=$ $10 \Delta t$, and is given as follows:

$$
\left[\begin{array}{llll}
\bar{u}_{j} & \bar{p} & \bar{\rho} & \bar{T}
\end{array}\right]^{R A N S}=\mathcal{C}_{L R}\left[\begin{array}{llll}
\left\langle\widetilde{u}_{j}\right\rangle_{\Delta T} & \langle\widetilde{p}\rangle_{\Delta T} & \langle\widetilde{\rho}\rangle_{\Delta T} & \langle\widetilde{T}\rangle_{\Delta T}
\end{array}\right]^{L E S}
$$

\subsection{Details of the Flow Solver}

The present framework is developed in the open-source computational fluid dynamics (CFD) software OpenFOAM $[47,26]$ and is based on cell-centred, finitevolume discretisation. Specifically, the rhoPimpleFoam solver is used in the present work to develop the embedding strategy. A schematic of the embedded LES RANS domain is shown in figure 1. As shown in this figure, grids of different density are employed in each of the RANS and LES regions. The non-conformal interface that arises because of this is handled with the existing arbitrary mesh interface (AMI) method in OpenFOAM. Synthetic turbulence generation is applied on the cells adjacent to the LES inflow interface before the fence as shown in the figure. Second-order accurate central difference schemes are used for discretisation in both the regions and the Crank-Nicolson scheme is used for temporal discretisation. The interface between RANS and LES is actually two interfaces, $\Gamma_{\text {RANS }}$ attached to the RANS domain $\left(\Omega_{\mathrm{RANS}}\right)$ and $\Gamma_{\mathrm{LES}}$ attached to the LES domain $\left(\Omega_{\mathrm{LES}}\right)$. Representing the interpolation operator by $\mathcal{I}_{R L}$ that interpolates data from $\Gamma_{\mathrm{RANS}}$ to $\Gamma_{\mathrm{LES}}$ and the coarsening operator by $\mathcal{C}_{L R}$ that restricts the data from $\Gamma_{\text {LES }}$ to $\Gamma_{\text {RANS }}$ we can express the overall algorithm in the following steps,

1. Update $\Gamma_{\text {RANS }}$ boundary conditions using $\mathcal{C}_{\mathrm{LR}}$

2. Solve for statistically averaged Navier-Stokes equations in $\Omega_{\mathrm{RANS}}$

3. Update $\Gamma_{\text {LES }}$ boundary conditions using $\mathcal{I}_{\mathrm{RL}}$

4. Solve for Favre-filtered Navier-Stokes equations in $\Omega_{\mathrm{LES}}$

5. Go to step 1.

Although the algorithm proceeds in a serial manner by solving the RANS and LES domains one after the other, the individual RANS and LES domains are 


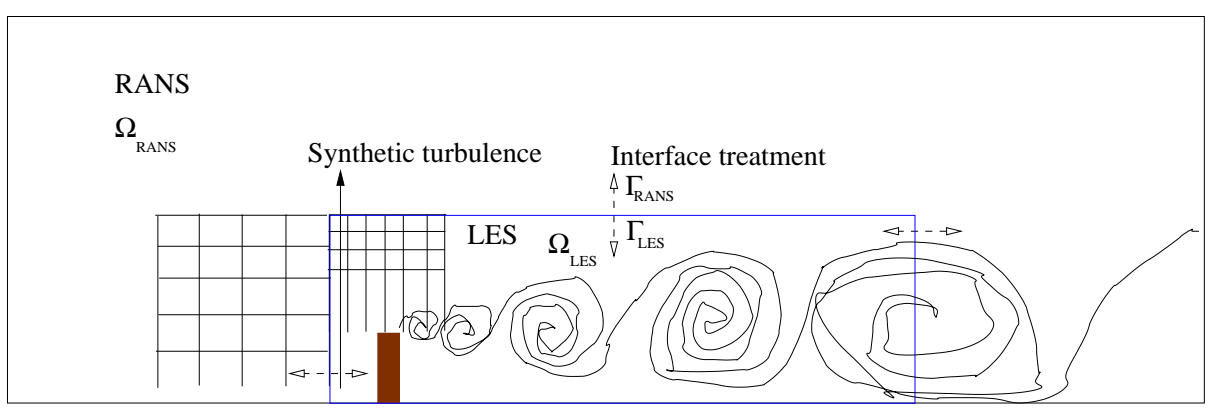

Fig. 1 Schematic of embedded LES region inside a global RANS region (not to scale).

solved in parallel. Also, it is hoped that in the limit of very coarse mesh in the RANS region the computational time will mainly be determined by the LES region. Such an explicit coupling of the interfaces is not new, e.g. several studies on gas turbine simulations have employed this strategy in the past $[39,21,40]$. However, coupling the interfaces within a single framework in an embedded sense by placing the interface close to the unsteady regions of the flow is novel and what the present work tries to accomplish.

\section{Results}

\subsection{Turbulent Channel Flow}

In order to validate the developed solver, we simulated spatially developing turbulent channel flow. The half height of the channel, $\delta$, is taken as the length scale and the friction velocity, $u_{\tau}$, is taken as the velocity scale to define the friction Reynolds number $R e_{\tau}=\rho u_{\tau} \delta / \mu$. In the present simulations we consider $R e_{\tau}=395$ for which [23] DNS data was available. A sketch of the zonal RANS-LES computational domain used is shown in figure 2. The domain size of the RANS channel is $L_{x 1}=15 \delta, L_{y}=2 \delta$ and $L_{z}=3.5 \delta$ in the streamwise $(x)$, wall-normal $(y)$ and spanwise $(z)$ directions, respectively. The size of the trailing LES channel is the same as the RANS channel except in the stream-wise direction which is $L_{x 2}=45 \delta$. The number of grid points for the RANS channel was $150 \times 80 \times 70$ and that in the LES channel was $450 \times 80 \times 70$ in the $x, y$ and $z$ directions, respectively. A uniform mesh was used in the streamwise and spanwise directions whereas a stretched mesh that clusters cells close to the top and bottom walls was used in the wallnormal direction. The centre of the first cell away from the walls was positioned such that $y^{+}=1$. The grid resolutions in wall-units in the $x$ and $z$ directions were $\Delta x^{+}=39.5$ and $\Delta z^{+}=19.8$.

As the Reynolds number of the channel flow problem was low the $k-\omega$ SST model was very diffusive and it was not feeding the right incoming turbulent boundary layer profile to the downstream LES region. As the RANS model is expected to provide a boundary condition for the scale-resolving region that is as accurate as possible, the low-Re version of the $k-\epsilon$ model by Launder and Sharma [16] was used, only for this particular case, instead as it was providing the 
correct incoming profiles to the LES region. In the LES region Smagorinsky [41] sub-grid scale model was used with van-Driest damping [45] with the Smagorinsky constant $C_{s}=0.065$. The inlet specification to the RANS channel consisted of

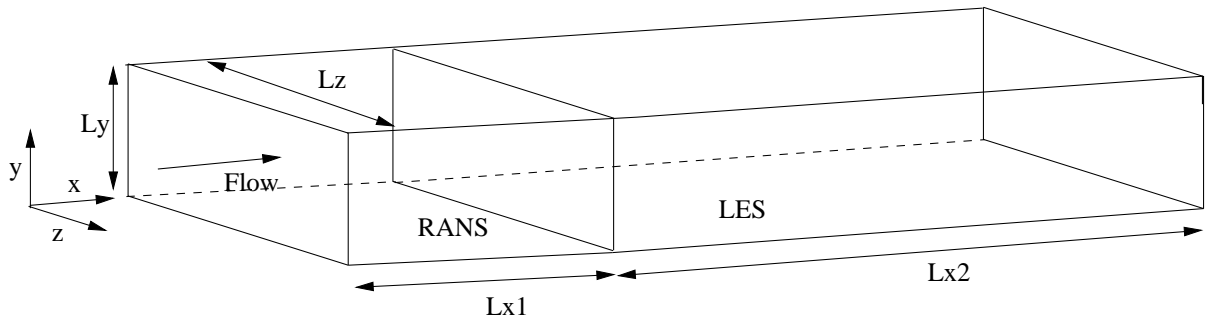

Fig. 2 A schematic of the zonal RANS - LES channel flow geometry (not to scale).

prescribing the mean velocity, $k$ and $\epsilon$ profiles as a function of the channel height $(y)$. These profiles were extracted from the DNS data base of Moser et al. [23]. At the RANS-LES interface, the digital filter based synthetic turbulence generator was used as described in the previous section. The integral length scales, nondimensionalised by $\delta$, in the streamwise direction were specified as $I_{11}=1, I_{21}=$ $I_{31}=0.5$ and in the spanwise direction as $I_{13}=I_{23}=0.16, I_{33}=0.2$. Further it was assumed that the integral length scales in the wall-normal direction were the same as that of those in the spanwise direction $\left(I_{i 2}=I_{i 3}\right)$. The study on digital filter method (by Kim et al. [9]) has tested and shown the sensitivity of the solution to the integral length scales to be small once a certain threshold is overcome; values following this guideline were chosen in the current study. At the LES inflow interface, the digital filter method used the mean velocity profiles from the upstream RANS region. Following an initialization period of $20 t^{*}$ statistics were collected for a period of $40 t^{*}$, where $t^{*}=t u_{\tau} / \delta$. A time step of $\Delta t^{*}=0.001$ was used which satisfies the condition that the Courant-Friedrichs -Lewy (CFL) number is less than unity. No-slip wall boundary conditions were applied on the top and bottom walls and a periodic boundary condition was applied in the spanwise direction.

The spatial variation of the non-dimensional wall shear stress, $\left\langle\tau_{w}\right\rangle$, is shown in figure 3. The wall-shear stress adjusts to a constant value within $x / \delta=2$ from the inlet in the RANS region and its value is maintained until it reaches the RANS-LES interface. At the interface, the wall-shear stress reaches a peak value owing to the DFM and thereafter it quickly drops to a minimum value and further downstream it recovers back reaching a constant value again by $x / \delta=35$. With the LES inflow interface positioned at $x / \delta=15$, the length of the channel for the wall-shear stress to reach a constant value is 20 . The time and spanwise averaged velocity in the streamwise direction is shown in figure 4(a) and the Reynolds stress components are shown in figure $4(\mathrm{~b})$. These profiles are extracted at $x / \delta=50$. The results obtained by Kim et al. [9] by using the same DFM but as an inflow boundary in a full LES simulation are also shown for comparison purposes. The mean velocity profile shows a good match with the [23] DNS data. The Reynolds stresses also show a satisfactory match with the DNS data. The streamwise component $\left\langle u^{\prime} u^{\prime}\right\rangle^{+}$is over estimated when compared with DNS whereas the wall-normal 


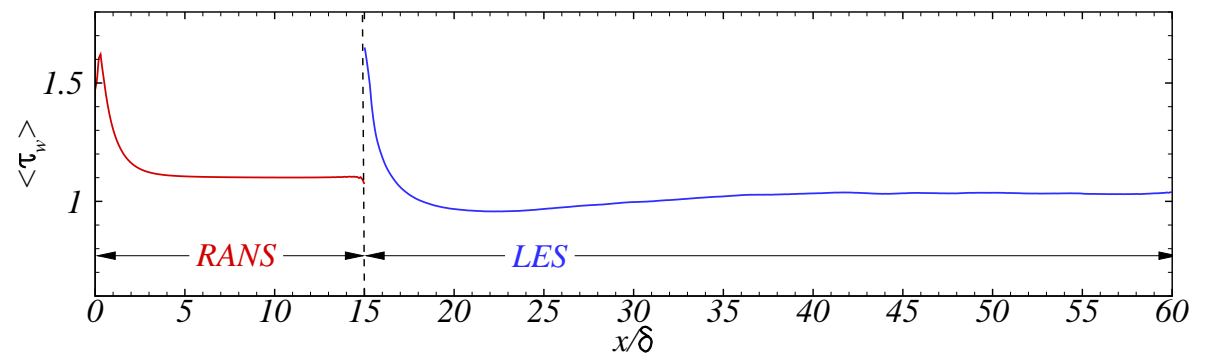

Fig. 3 Spatial variation of dimensionless wall shear stress, $\left\langle\tau_{w}\right\rangle$, along the channel.

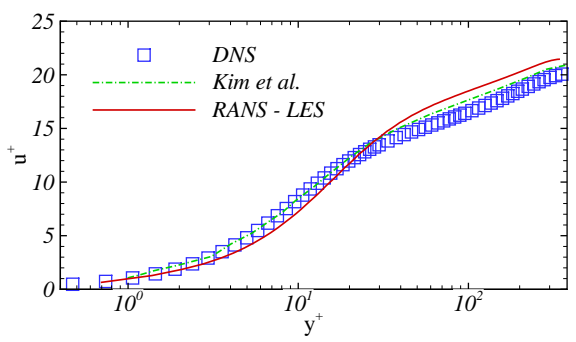

(a) Mean velocity

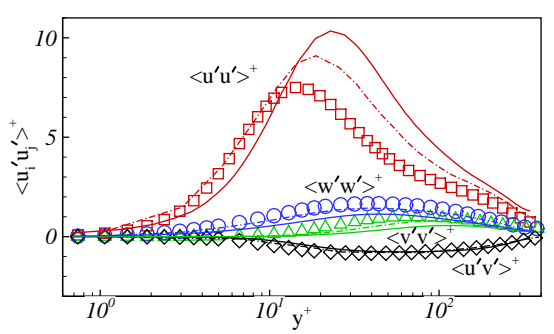

(b) Reynolds stresses

Fig. 4 Profiles of mean velocity and Reynolds stresses (solid lines), extracted at $x / \delta=50$, compared with the DNS data (symbols) and data obtained from LES simulations of Kim et al. [9] (dash dot lines).

$\left\langle v^{\prime} v^{\prime}\right\rangle^{+}$and spanwise components $\left\langle w^{\prime} w^{\prime}\right\rangle^{+}$are under estimated. The shear stress component $\left\langle u^{\prime} v^{\prime}\right\rangle^{+}$shows a satisfactory match with the DNS. Overall, the mean and Reynolds stress profiles from the present simulations agree closer to the LES data of Kim et al. [9] also using a DFM. To estimate the length required for the synthetic turbulence to reach realistic turbulence profiles the normal and shear components of Reynolds stresses are plotted, in outer co-ordinates, at several locations downstream of the LES interface in frames (a) and (b) of figure 5. From

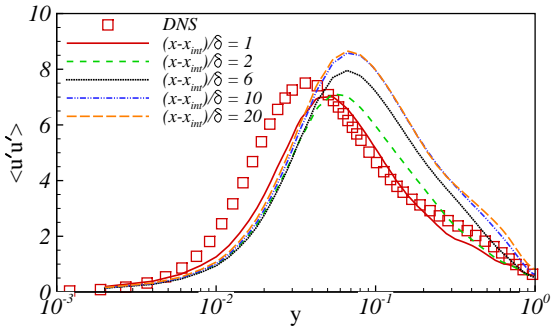

(a) $\left\langle u^{\prime} u^{\prime}\right\rangle$

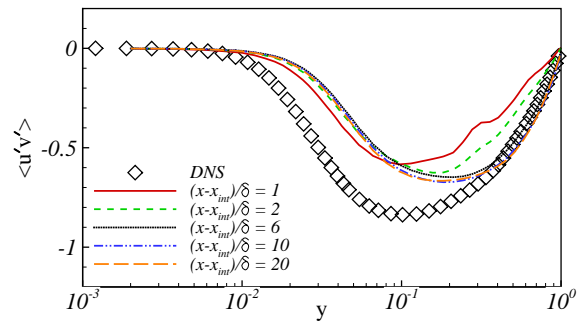

(b) $\left\langle u^{\prime} v^{\prime}\right\rangle$

Fig. 5 Profiles of Reynolds stress components, plotted in outer co-ordinates, at indicated locations downstream of the LES interface (lines) together with the DNS data (symbols). The LES interface $x_{i n t} / \delta=15$ 
this figure it can be seen that the normal stresses $\left(\left\langle u^{\prime} u^{\prime}\right\rangle\right)$ become self-similar from $\left(x-x_{\text {int }}\right) / \delta=10$ onwards. However, the shear stress profiles $\left(\left\langle u^{\prime} v^{\prime}\right\rangle\right)$ already become self-similar from $\left(x-x_{\text {int }}\right) / \delta=6$ onwards. As the shear stresses are thought to be more important than the normal stress components in this type of flow, a length of at least $6 \delta$ appears necessary in order to obtain profiles that represent real turbulence. In figure 3 it was noted that it takes nearly $20 \delta$ for the boundary layer to fully develop in terms of the skinfriction. However, the Reynolds stress profiles approached the desired value much earlier and this length is the one we used for the subsequent simulations, the argument being that we wanted reasonably realistic fluctuations with roughly the correct mean flow at the interface, but not accepting the excessive cost of having to resolve the synthetic turbulence that far upstream. To qualitatively visualise the effect of digital filtering on the flow structures $Q$-criterion can be used. The $Q$-criterion is defined as the second invariant of the velocity gradient tensor and given as follows, $Q=\frac{1}{2}\left(\Omega_{i j} \Omega_{i j}-S_{i j} S_{i j}\right)$, where $\Omega_{i j}=\frac{1}{2}\left(\frac{\partial u_{i}}{\partial x_{j}}-\frac{\partial u_{j}}{\partial x_{i}}\right)$ is the rotation rate and $S_{i j}=\frac{1}{2}\left(\frac{\partial u_{i}}{\partial x_{j}}+\frac{\partial u_{j}}{\partial x_{i}}\right)$ is the strain rate. Iso-surfaces of $Q=100 \mathrm{~s}^{-2}$ are shown in figure 6 . The $Q$ iso-surfaces

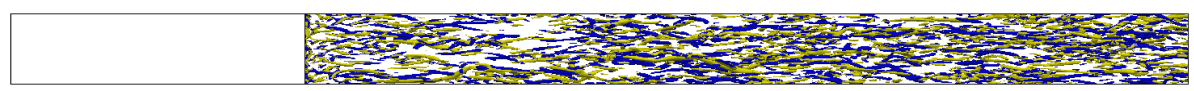

(a) $x z$-plane

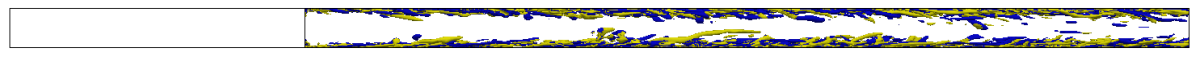

(b) $x y$-plane

Fig. 6 Iso-surfaces of $Q=100 \mathrm{~s}^{-2}$ coloured by instantaneous streamwise vorticity, positive values (yellow) and negative values (blue) for the channel flow problem solved using RANSLES.

are coloured by positive (yellow) and negative (blue) streamwise vorticity values. It can be noted that streamwise vorticity values occur in pairs on most of the $Q$ iso-surfaces. The coherent structures fed by DFM are relatively sparse just downstream of the RANS-LES interface but are in abundance further downstream. Through this test case we achieved reasonably realistic turbulent fluctuations, but not a perfect channel simulation. However, in the present work the main objective was to get fluctuations after a reasonably short development length that are approximating real turbulent fluctuations so that the LES domain can produce the correct mean flow. As it is showing good performance it will now be used for more complex applications.

\subsection{Flow Over a Surface-Mounted Vertical Fence}

\subsubsection{Problem Setup and Boundary Conditions}

The problem considered here is flow over a surface-mounted vertical fence configuration for which DNS results exist in the literature [27]. The height of the fence 
$(h)$ is taken as the length scale and the maximum velocity $(U)$ of the incoming turbulent boundary layer is taken as the velocity scale to define the Reynolds number, $R e=\rho U h / \mu$, which is equal to 3000. A schematic of the embedded LESRANS domain for the flow over the vertical fence is shown in figure 1 . At the inlet of the RANS domain, a turbulent boundary layer profile is prescribed together with the turbulent kinetic energy profile as used in the DNS [27]. At the far-field boundary a slip boundary condition on the velocity is specified. At the outlet of the RANS domain a zero-gradient boundary condition on velocity is prescribed and non-reflective boundary conditions on pressure are applied. No-slip boundary conditions are applied on the bottom wall including the fence boundaries. A time step of 0.001 is utilised for all the simulations and this satisfies the condition that CFL is less than unity. The domain size of the individual RANS and LES regions together with their mesh resolutions used for the two cases considered are presented in table 1 . The mesh resolution used in the DNS simulation by Orellano \& Wengle [27] is also tabulated for comparison purposes. Primarily two cases are considered, with the LES interface placed at $3 h$ and $6 h$ upstream of the fence. The case with no synthetic turbulence was run on the same mesh as that of the $3 h$ LES interface case. In the cases with synthetic turbulence at the LES interface, DFM was used with both mean velocity and Reynolds stresses extracted from the upstream RANS interface. The integral length scales, non-dimensionalised by $h$, in the streamwise direction were taken as $I_{11}=1, I_{21}=0.5, I_{31}=0.5$ and in the wall-normal direction as $I_{12}=I_{22}=I_{32}=0.3$ in the outer region $(y / h>0.1)$ and in the inner region $(y / h<=0.1)$ they were taken as $I_{12}=I_{22}=I_{32}=0.15$. Further, the integral length scales in the spanwise direction are assumed to be the same as those in the wall-normal direction $\left(I_{i 3}=I_{i 2}\right)$. Each of the simulations were first run for an initialisation period of $180 t^{*}$ after which the synthetic turbulence generation was switched on at the LES interface. Following this initialisation period, the mean and turbulent statistics were collected for another $300 t^{*}$. In order to qualitatively visualise the vortical structures in the LES region, iso-surfaces of $Q=0.5 \mathrm{~s}^{-2}$ are shown in figure 7 . The colours on these iso-surfaces indicate positive streamwise component of vorticity (yellow) and negative streamwise component of vorticity (blue). These structures are absent in the RANS region. Vortical structures fed at the LES interface are noticed and develop into larger structures downstream of the fence.

Table 1 Details of the domain and mesh resolution used for each of the vertical fence simulation cases

\begin{tabular}{lllll}
\hline Case & Domain & & Mesh & Total Mesh \\
\hline & RANS & LES & RANS & LES \\
\hline 1. LES interface at $-3 h$ & $(35 h, 8 h, 5 h)$ & $(19 h, 5 h, 5 h)$ & $0.547 \times 10^{6}$ & $1.54 \times 10^{6}$ \\
$x$-direction & $-10 h$ to $-3 h, 16 h$ to $25 h$ & $-3 h$ to $16 h$ & & \\
-direction & 0 to $8 h, 5 h$ to $8 h$ & 0 to $5 h$ & & \\
z-direction & 0 to $5 h$ & 0 to $5 h$ & & \\
2. LES interface at $-6 h$ & $(40 h, 8 h, 5 h)$ & $(22 h, 5 h, 5 h)$ & $0.582 \times 10^{6}$ & $1.924 \times 10^{6}$ \\
$\quad x$-direction & $-15 h$ to $-6 h, 16 h$ to $25 h$ & $-6 h$ to $16 h$ & & \\
$y$-direction & 0 to $8 h, 5 h$ to $8 h$ & 0 to $5 h$ & & \\
$z$-direction & 0 to $5 h$ & 0 to $5 h$ & & - \\
3. DNS data $[27]$ & $(39 h, 6 h, 5 h)$ & - & & \\
\hline
\end{tabular}




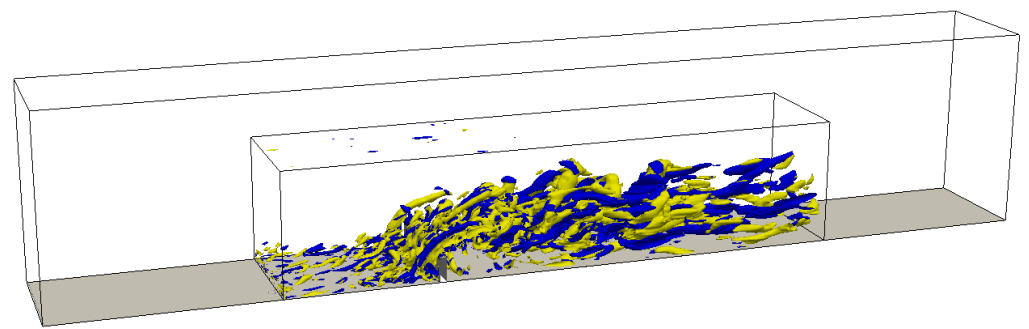

Fig. 7 Iso-surface of $Q=0.5 \mathrm{~s}^{-2}$ coloured by instantaneous streamwise vorticity, positive values (yellow) and negative values (blue), for the vertical fence case solved using embedded LES-RANS

\subsubsection{Case with No Synthetic Turbulence Generation at the LES Interface}

First, in order to understand the need and the importance of providing turbulence fluctuations at the LES interface we simulated a case with no synthetic turbulence generation at the interface and with full coupling of the flow and turbulence quantities. The results obtained from this simulation are shown in figure 8 by dash-dot lines (blue in colour) and the DNS data is shown by hollow circle symbols in all of the frames. The normalised mean streamwise velocity, as shown in frame (a) of this figure, does not match with the DNS data upstream of the fence because without adding fluctuations to the LES region the flow relaminarises resulting in a taller re-circulation zone (at $x / h=-0.87$ ). The profiles extracted on the fence and further downstream at locations $x / h=0.25,1.5,2.75,5.25$ deviate from the DNS result for $y / h \leq 2$. Similar observations can be made for the normalised mean wall-normal component of velocity in frame (b). Comparing the second-order statistics, we can more clearly distinguish the differences between embedded LESRANS with no synthetic turbulence case and the DNS data. The root-mean-square streamwise velocity fluctuations, as shown in frame (c), are absent at all the wallnormal locations upstream of the fence at $x / h=-2.3,-0.87$ and its peak values are largely underestimated at locations on the fence and locations just downstream $(x / h=0.25,1.5)$. The $u_{r m s} / U$ values reach the same peak value as the DNS data at $x / h=2.75$ and are overestimated for the rest of the downstream locations. The in-plane Reynolds stress component shown in frame(d) follows a similar trend as $u_{r m s}$ and by connecting the peak values of all the profiles a different trajectory, for the shear flow emanating from the top of the fence, can be perceived than that of the DNS data. The results obtained from this case with no synthetic turbulence generation clearly indicate that both the mean quantities and second moments show differences, the latter to a larger extent, in comparison to the DNS data, and emphasise the need to feed turbulent fluctuations at the LES interface.

\subsubsection{Effect of the Location of the LES Interface}

Next, we consider simulations by introducing turbulence fluctuations at the LES interface. As the synthetic fluctuations require a certain development length to transition the flow into real turbulence in the LES region, as shown for the channel 


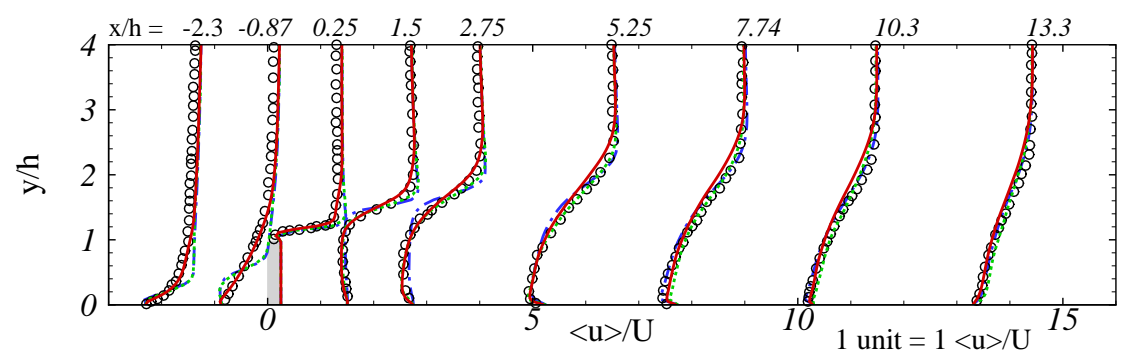

(a) $\langle u\rangle / U$

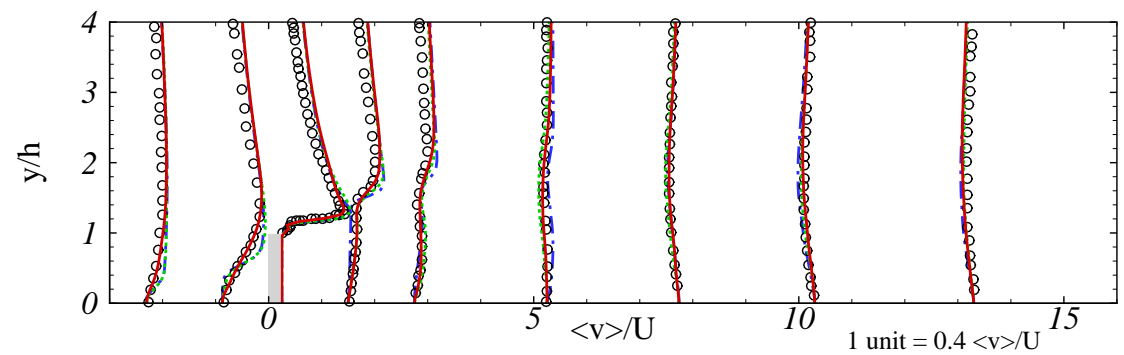

(b) $\langle v\rangle / U$

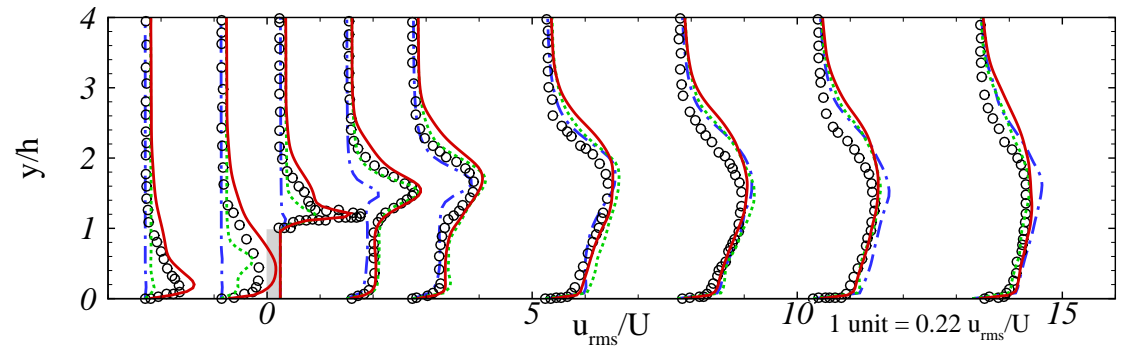

(c) $u_{r m s} / U$

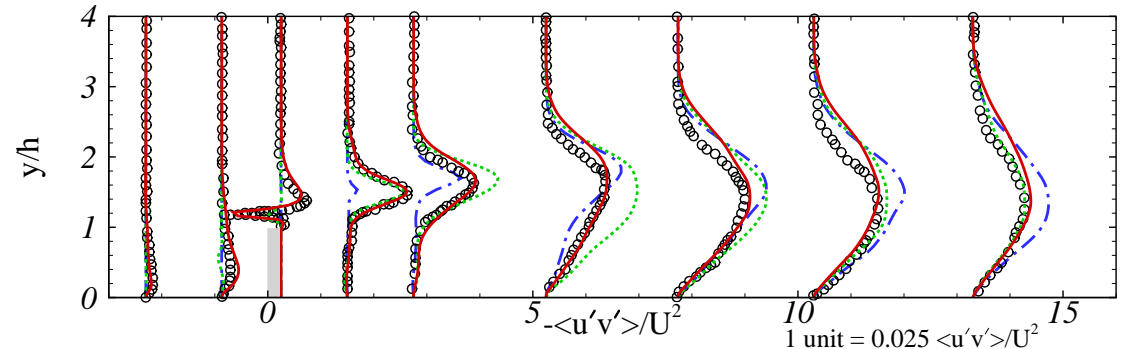

(d) $-\left\langle u^{\prime} v^{\prime}\right\rangle / U^{2}$

Fig. 8 Mean and second-order statistics for DNS [27] (symbols), embedded LES-RANS with no synthetic turbulence (dash-dot line in blue), with synthetic turbulence and LES interface placed at three fence-heights upstream of the fence (dash line in green), with synthetic turbulence and LES interface placed at six fence-heights upstream of the fence (solid line in red). 
flow case in section 3.1, we considered two cases. In the first case, the LES interface was placed at three fence-heights upstream of the fence. In the second case, it was placed six fence-heights upstream of the fence. This value was guided by looking at how quickly the Reynolds stress components developed to a self-similar turbulent profile in the channel flow case of section 3.1. The results obtained from both the simulations are shown in figure 8 , by green coloured dash lines for the case with the LES interface at three fence-heights, by red coloured solid lines for the case with the LES interface placed at six fence-heights upstream of the fence. From the frames (a) and (b) for the streamwise and wall-normal velocity profiles it can be seen that the results obtained from the case with three fence-heights coincide with that of the case with no synthetic turbulence (dash dot blue lines) at locations upstream and on the fence. However, unlike the case with no synthetic turbulence generation, the three fence-height LES interface case results agree better with the DNS results for the mean flow quantities from $x / h=1.5$ onward. Looking at the three fenceheights interface case for the second-order statistics we observe that the $u_{r m s} / U$ profiles are non zero upstream of the fence and appear to be developing with the DNS data as the target values. A qualitatively similar profile with peak values underestimated can be observed upstream and on the fence locations in frames (c) and (d). Even with underestimated peak values right on the fence, surprisingly at the very next location $(x / h=1.5)$ both $u_{r m s}$ and $-\left\langle u^{\prime} v^{\prime}\right\rangle$ profiles match well with the DNS data, however they begin to deviate further downstream, with the in-plane Reynolds stresses showing a greater deviation. Therefore, the three fenceheights LES interface case suggests that the LES interface should be moved further upstream to allow for the turbulent stresses to develop fully before reaching the fence. Finally, we present the results obtained from the case with the LES interface placed at six fence-heights upstream of the fence. The profiles are plotted using solid lines coloured in red in all the frames in figure 8 . The mean velocity profiles in frames (a) and (b) match well with the DNS data at all the streamwise locations. The second-order statistics in frames (c) and (d) also show good agreement with the DNS data at all the locations. This case indicates that placement of the LES interface, when the turbulence fluctuations are imposed, plays a critical role. In the present case, placing the LES interface at six fence-heights is observed to be sufficient to yield matching results with the DNS data.

\subsubsection{Streamwise Variation of Wall Shear Stress}

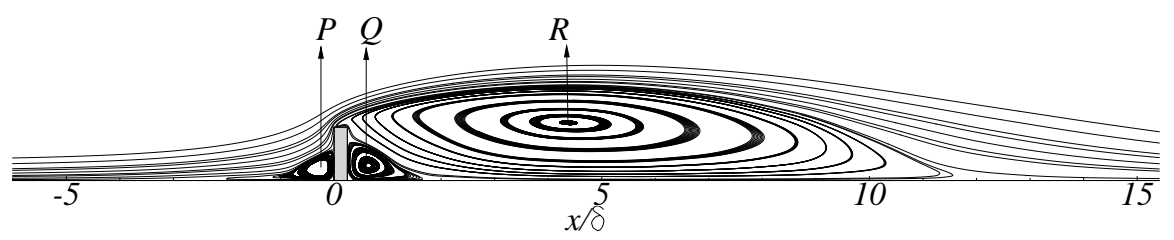

Fig. 9 Streamlines on the $x y$-plane, computed using mean and spanwise averaged velocity components, depicting three recirculation regions for the case with the LES interface at $-6 h$.

The mean spanwise averaged $x y$-plane velocities are used to generate streamlines and are shown in figure 9 for the case with the LES interface at $-6 h$. Three 
recirculation regions can be seen, one before the fence $(\mathrm{P})$, one immediately after the fence $(\mathrm{Q})$ and a primary recirculation bubble behind the fence $(\mathrm{R})$. To quantify the length of each of these recirculation bubbles we plot the variation of wall shear stress on the bottom wall. The streamwise variation of wall skin friction is computed for all the cases with an embedded LES region and is shown in figure 10. The DNS data are plotted using hollow circle symbols. The values of the mean separation location for all the cases are presented in table 2 . The case with no fluctuations added and the case with the LES interface at $-3 h$ follow a similar profile upstream of the fence. The mean separation length of the recirculation bubble before the fence extends further upstream into the RANS region $\left(x_{P} / h=-3.8\right)$ for the case with the interface at $-3 h$. The reason for this long recirculation bubble is that the flow relaminarises before it reaches the fence as a consequence of the synthetic turbulence fed at the interface still developing. A closer agreement of the mean separation location with the reference data $\left(x_{P} / h=-2.0\right)$ is observed for the case with the LES interface at $-6 h\left(x_{P} / h=-2.8\right)$. The location of separation of the secondary recirculation bubble immediately behind the fence is overestimated by the case with the LES interface at $-3 h\left(x_{Q} / h=2.9\right)$ and the case with no fluctuations added $\left(x_{Q} / h=3.9\right)$ when compared with the reference data $\left(x_{Q} / h=2.6\right)$. The case with the LES interface at $-6 h$ under-estimates this location $\left(x_{Q} / h=1.8\right)$, however, it shows a better agreement for the location of the primary recirculation bubble $\left(x_{R} / h=13.0\right)$ when compared with the reference data $\left(x_{R} / h=13.2\right)$. The cases with the LES interface at $-3 h$ and no fluctuations added under-estimate this location, $x_{R} / h=11.0$ and $x_{R} / h=12.6$, respectively. The case with LES interface at $-6 h$ shows a peak in wall shear stress just upstream of the fence which is qualitatively similar to that observed in the DNS data at a higher Reynolds number. Overall, we observe that the case with the LES interface at $-6 h$ shows better agreement for the location of the primary recirculation bubble before and after the fence.

Table 2 Mean separation location for the recirculation bubble for all the cases

\begin{tabular}{llll}
\hline Case & $x_{P} / h$ & $x_{Q} / h$ & $x_{R} / h$ \\
\hline 1. DNS data [27] & -2.0 & 2.6 & 13.2 \\
2. LES interface at $-6 h$ & -2.8 & 1.8 & 13.0 \\
3. LES interface at $-3 h$ & -3.8 & 2.9 & 11.0 \\
4. No synthetic turbulence generation & -3.8 & 3.9 & 12.6 \\
\hline
\end{tabular}

\subsection{Flow Over a Surface-Mounted Inclined Spoiler}

In this section, we apply the developed method to solve flow over an inclined spoiler. Unlike the previously studied fence case the spoiler has a finite width in the spanwise direction and introduces three-dimensionality into the problem. The spoiler is inclined at 30 degrees to the streamwise direction. Nedić et al. [25] performed particle image velocimetry and force measurements for the inclined spoiler case and their results will be used as the reference to compare the present simulations. 


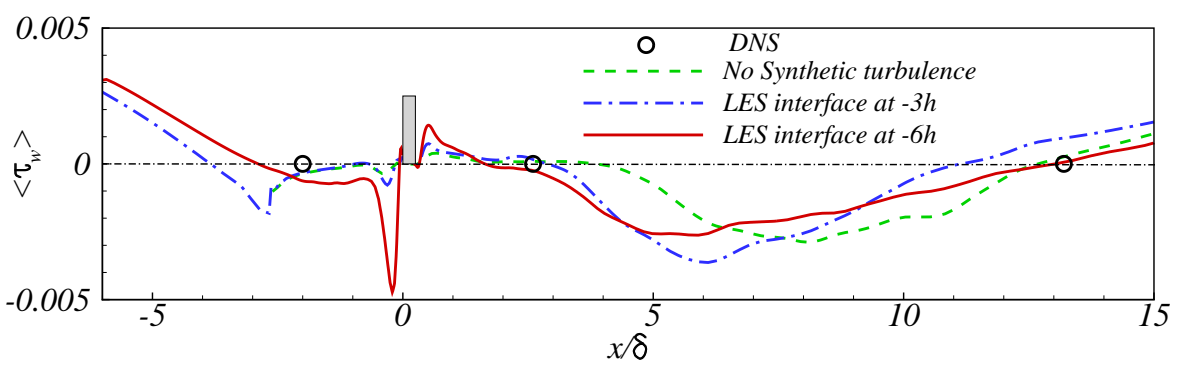

Fig. 10 Variation of wall skin friction along the bottom wall for all the embedded LES-RANS cases and the DNS data [27].

\subsubsection{Problem Setup and Boundary Conditions}

A schematic of the inclined spoiler configuration is shown in figure 11. The domain size extends $10 m \times 0.5 m \times 1 m$ in the streamwise, wall-normal and spanwise directions respectively. The spoiler is $106 \mathrm{~mm}$ long, $247 \mathrm{~mm}$ wide and $4 \mathrm{~mm}$ thick and the inlet velocity of the flow is $40 \mathrm{~m} \mathrm{~s}^{-1}$. Taking the spoiler length $(h)$ and inlet velocity of the flow as the length and velocity scales respectively and by specifying the air density and viscosity at the inlet as $1.16 \mathrm{~kg} \mathrm{~m}^{-3}$ and $1.84 \times 10^{-5} \mathrm{~Pa} \mathrm{~s}^{-1}$ the inlet Reynolds number of the flow can be calculated as $2.67 \times 10^{5}$. As the details of the incoming boundary layer profile (mean velocity profile upstream of the fence) are not available from the experiments, the leading edge of the wind-tunnel with the same length as that in the experiments is considered in the simulations by specifying a uniform inlet velocity profile and letting a turbulent boundary layer develop using a RANS model. In fact, this test case therefore is a good example for the benefits the embedding strategy offers, as an LES of the entire wind tunnel set-up would not have been feasible. The LES interface was located at $6 h$ upstream of the base of the inclined spoiler. The total mesh resolution used is

$94.3 \mathrm{~h}$

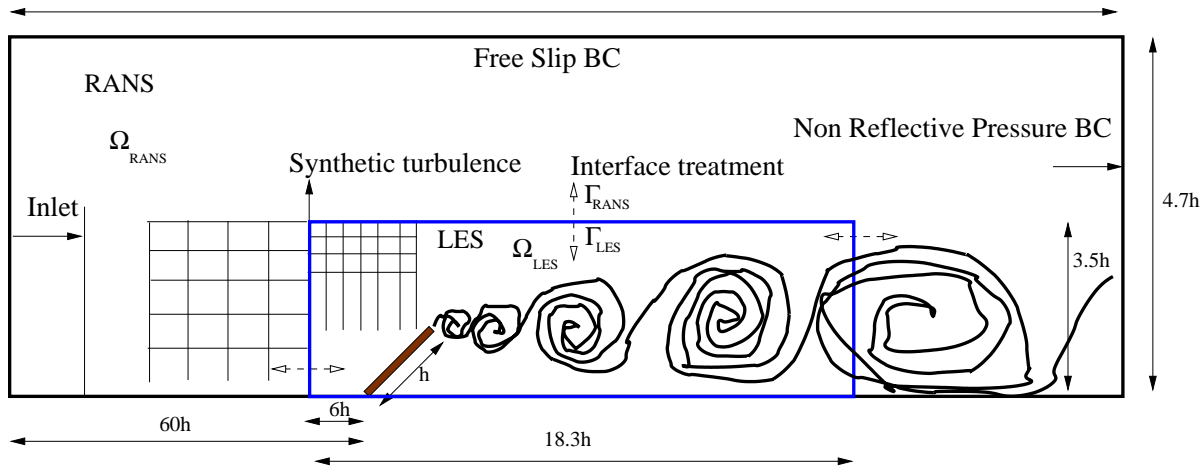

Fig. 11 Schematic of the embedded LES region inside a global RANS region for the flow over inclined spoiler (not to scale). 
2 million cells with 1.8 million cells in the embedded LES region and 0.28 million cells in the RANS region. At the far-field boundary a slip boundary condition on velocity is specified and non-reflective boundary conditions are prescribed for pressure. Unlike the previous case, the spoiler in the present case is of finite-width, therefore symmetry boundary conditions are applied on the spanwise boundaries. No-slip velocity boundary condition is applied on the bottom surface including the spoiler geometry. A time step of $10^{-6}$ seconds is used for the present case and it satisfies the condition that CFL is less than unity. The integral length scales, non-dimensionalised by the length of the spoiler, in the streamwise direction were taken as $I_{11}=1, I_{21}=0.5, I_{31}=0.5$ and in the wall-normal direction as $I_{12}=I_{22}=I_{32}=0.5$ in the outer region $(y / h>0.1)$ and $I_{12}=I_{22}=I_{32}=0.25$ in the inner region $(y / h<=0.1)$. Owing to the lack of information about the incoming turbulent integral length scales, the incoming turbulent boundary layer was considered as a wall-bounded flow and the spanwise integral length scales were taken to be the same as those in the wall-normal direction, as was done in the channel flow case. After an initialisation period of 2 flow through times, equivalent to $160 t^{*}$, the feeding of synthetic fluctuations at the LES inflow interface was switched on and the statistics were collected for 3 flow through times, equivalent to $240 t^{*}$ where $t^{*}$ is the time scale based on the length of the spoiler and the inlet velocity.

\subsubsection{Comparison with the Experiments}

The mean and second-order statistics are now compared with the experimental data [25]. The normalised streamwise velocity is shown in frame (a) of figure 12 at the indicated locations together with the experimental data. The measurements as well as the profiles from the present simulations are extracted on a plane that is $82 \mathrm{~mm}$ from the edge of the spoiler and parallel to the center plane. In the present case, the spanwise direction is not homogeneous and hence all the profiles are only time averaged. As can be seen from this figure a very good match with the experimental data is obtained at the locations $x / h=-0.1,0.84$ and 1.6. The velocity profile at $x / h=-0.1$ shows a taller recirculation bubble than in the experiments. A comparison of $u_{r m s}$ values is presented in frame (b) of this figure. Overall, a good comparison is obtained with respect to the experiments. The extent of the $u_{r m s}$ is captured at $x / h=-0.1$ with a slightly larger peak. The profiles follow those of the experiments at the other two locations. At the tip of the spoiler, the strong fluctuations that exists in the experiments could not be captured by the present simulation, albeit it is not clear whether the measurements are accurate at this location or not. The growth of peak values of $u_{r m s}$ can be seen from $x / h=-5$ onwards because of the synthetic fluctuations introduced further upstream. Next, the profiles of $\langle u\rangle$ and $u_{r m s}$ are compared in the $x z$-plane that is parallel to the bottom wall and at a height of $24 \mathrm{~mm}$ above it. Frames (c) and (d) of this figure show profiles of $\langle u\rangle$ and $u_{r m s}$ respectively. As can be seen from these figures a very good match with the reference data is obtained at all the indicated locations. 


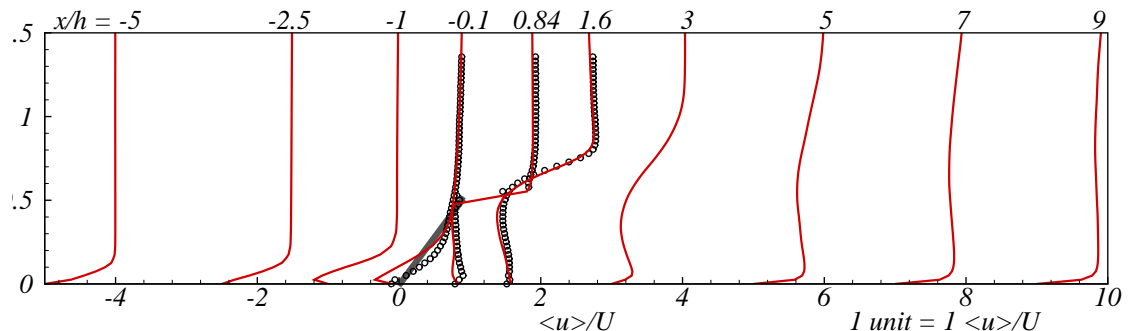

(a) $\langle u\rangle / U$

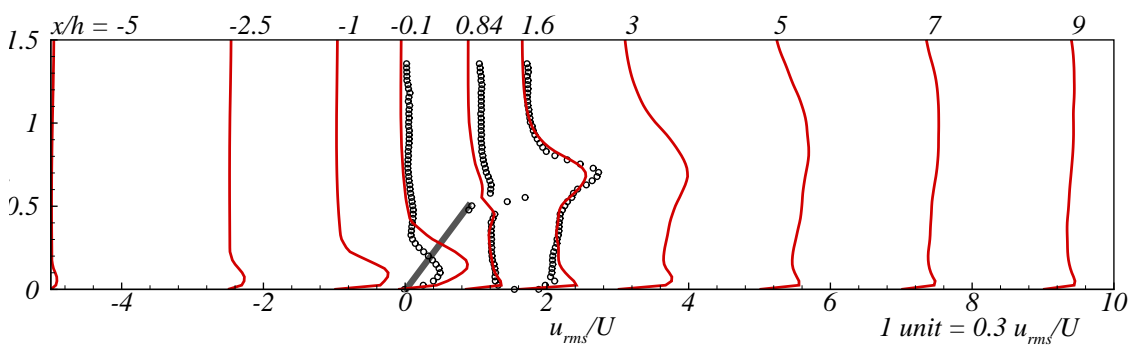

(b) $u_{r m s} / U$

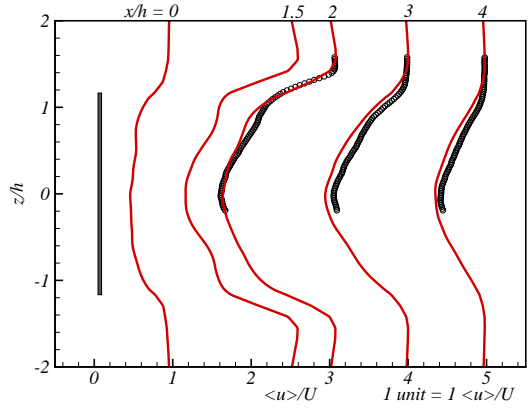

(c) $\langle u\rangle / U$

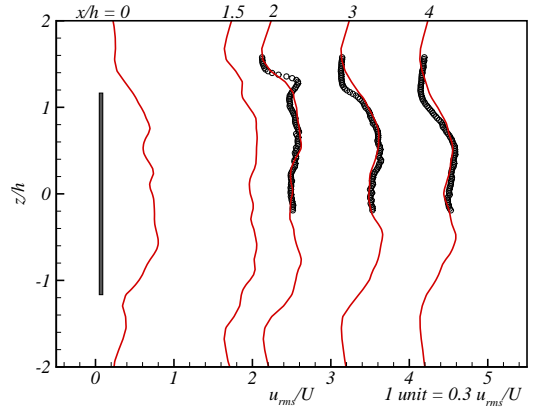

(d) $u_{r m s} / U$

Fig. 12 Mean and second-order statistics for experiments [25] (symbols) and embedded LESRANS (solid lines in red). Frames (a) and (b) show profiles on $x y$-plane and (c) and (d) denote them on $x z$ plane at $24 \mathrm{~mm}$ away from the bottom plate.

\subsection{Flow Over an Open Cavity}

Flow over an open cavity is encountered in aerospace applications such as weaponsbays. The shear layer developing from the fore-wall creates pressure oscillations that reflect after hitting the aft-wall of the cavity. These pressure oscillations generate both broadband and tonal noise. Flow over open cavities has been extensively studied in the literature because of its practical application and the rich flow physics it generates. Several studies in the past have used experimental $[35,13$, $31,11,36,7]$ or computational techniques $[5,15,37,17]$ to understand the physics of these flows. Rossiter [36] developed a simple expression to calculate the frequencies of the pressure tones in the spectra by performing experiments and analysing the 
experimental data from cavities with varying aspect ratio over a range of Mach numbers. Henshaw [7] provided pressure spectra and root-mean-square pressures obtained from experiments for flow over cavities with Mach numbers ranging from 0.4 to 1.19. Larchevêque et al. [14] performed LES simulations for the open cavity case at a Mach number of 0.85 and compared the results to the experimental data provided by Henshaw [7]. Lawson \& Barakos [17] performed DES of weaponsbay geometries and analysed the flow. Recently, Kim et al. [8] performed DES of weapons bay flows and store separation and concluded that by steady blowing the pressure fluctuations can be mitigated. A review of numerical simulations applied to open cavity flows can be found in the article by Lawson \& Barakos [18] and Grace [6] gives an overview of computational aeroacoustic techniques applied to open cavity noise. In the present work, owing to the relevance to the case, we choose the experimental data by Henshaw [7] and the LES data by Larchevêque et al. [14] as the reference data.

\subsubsection{Problem Setup and Boundary Conditions}

A schematic of the embedded LES-RANS domain used for solving the flow over an open cavity is shown in figure 13 . The cavity has an aspect ratio of $5 \times 1 \times 1$ in the streamwise, wall-normal and spanwise directions respectively. The configuration is the same as the one considered in the experimental measurements [7] and in the LES simulation of Larchevêque [14]. The depth of the cavity $(D)$ equals $0.1016 \mathrm{~m}$.

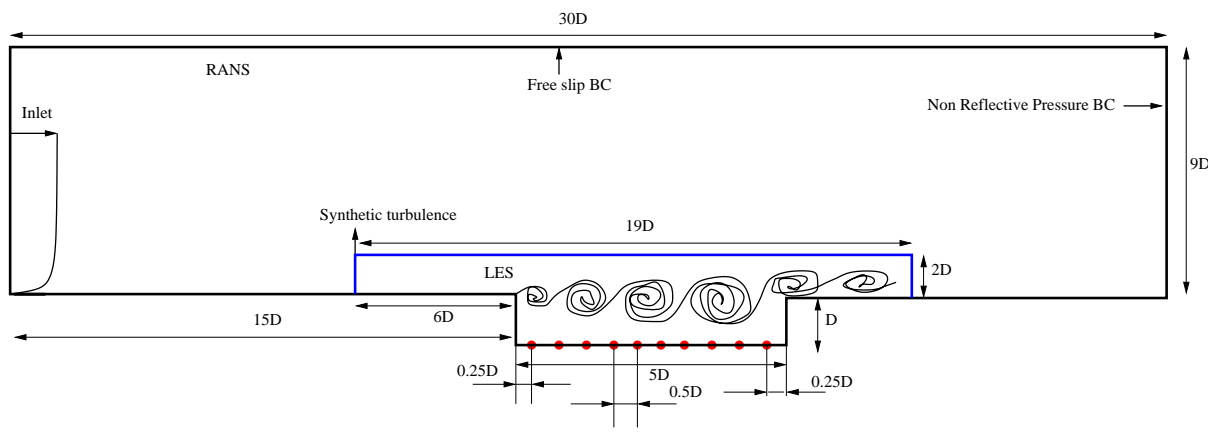

Fig. 13 Schematic of the flow over open cavity as an embedded LES - RANS domain (not to scale). The red circles indicate the pressure recording locations on the cavity floor that are spaced equally with a gap of $0.5 D$ and the first pressure recorder is at a distance of $0.25 D$ from the fore-wall of the cavity.

The LES domain extends $19 D \times 3 D \times 8 D$ and the RANS domain is $30 D \times 9 D \times 8 D$ in the streamwise, wall-normal and spanwise directions. The inflow velocity is $276.54 \mathrm{~ms}^{-1}$, the stagnation pressure and temperature are $99540 \mathrm{~Pa}$ and $301 \mathrm{~K}$ respectively. The Reynolds number of the flow based on the length of the cavity and the inflow velocity is $7 \times 10^{6}$ and the inflow Mach number equals 0.85 . A total mesh resolution of 7.6 million cells was used with 6.5 million cells in the LES region and 1.1 million cells in the RANS region. The number of cells only in the cavity were 2.5 million. A time step of $10^{-6}$ seconds was used for all the simulations and the first 
0.1 seconds was considered as the initial transient and the turbulence statistics were collected for 0.5 seconds thereafter. For the synthetic turbulence generation, the integral length scales non-dimensionalised by the boundary layer thickness $\left(\delta_{99}\right)$, in the streamwise direction were taken as $I_{11}=1, I_{21}=0.5, I_{31}=0.5$ and in the wall-normal direction as $I_{12}=I_{22}=I_{32}=0.5$ in the outer region $(y / D>0.1)$ and $I_{12}=I_{22}=I_{32}=0.25$ in the inner region $(y / D<=0.1)$. The integral length scales in the spanwise direction were equal to those in the wall-normal direction. To analyse the pressure field recorded by the probes on the floor of the cavity we define the following quantities. The power spectral density (PSD) of pressure fluctuations can be calculated using fast Fourier transform (FFT), however, owing to the short period of the recorded signal $(0.5 \mathrm{sec})$ we use Burg's estimator similar to the previous studies [14]. The PSD is useful to identify the frequencies at which pressure peaks occur that are responsible for the tonal noise. From the PSD, one can define the sound pressure level (SPL) which is a measure of perceived sound level in decibels $(\mathrm{dB})$ as follows

$$
S P L(f)=10 \log _{10}\left[\frac{P S D(f) \Delta f_{r e f}}{p_{r e f}^{2}}\right],
$$

where $\Delta f_{\text {ref }}$ is the reference frequency spacing usually taken as $1 \mathrm{~Hz}$ and $p_{\text {ref }}$ is the reference pressure, corresponding to minimum audible sound, taken as $20 \mu P a$. In order to obtain the global contribution of the tones, the SPL can be integrated over a prescribed range of frequency bands that contain one tone in each of them, this quantity known as band-integrated sound pressure level (BISPL) is defined as follows

$$
B I S P L=10 \log _{10}\left[\left(\int_{f_{1}}^{f_{2}} P S D(f) d f\right) \frac{1}{p_{r e f}^{2}}\right],
$$

where $f_{1}$ and $f_{2}$ are prescribed frequency bounds of integration. Further, the rootmean-square pressure fluctuations can be calculated as follows

$$
p_{r m s}^{\prime}=\sqrt{\frac{1}{N} \sum\left(p^{\prime}\right)^{2}},
$$

where $N$ is the length of the pressure fluctuation signal. An overall sound pressure level (OASPL) can be obtained from the $p_{r m s}$ as

$$
O A S P L=20 \log _{10}\left[\frac{p_{r m s}^{\prime}}{p_{r e f}}\right]
$$

\subsubsection{Comparison with the Reference Data}

In this section, we present comparisons of mean flow variables, second-order statistics and SPL to the reference LES [14] and the reference experiments [7]. A comparison of normalised streamwise and wall-normal velocities on the center plane of the cavity are shown in frames (a) and (b) of figure 14 (solid lines in red). A good match with the reference data is obtained at most of the locations along the cavity. Streamwise velocity profiles show a small disagreement at $x / D=3,3.5,4$ whereas the wall-normal velocities shows a slight disagreement at $x / D=3,3.5$. Normalised Reynolds shear stress and turbulent kinetic energy on the center plane 
of the cavity are shown in frames (a) and (b) of figure 15 (solid lines in red). A good match with the reference data can be seen at all the locations along the cavity. A small disagreement is observed at $x / D=3,3.5$ and 4 in both shear stress and turbulent kinetic energy profiles. Next, we analyse the pressure fluctuations on the cavity floor. Several pressure transducers were placed in the experiment [7] (K20 through K29) off-center from the cavity center plane to record the pressure fluctuations. In the present simulations, we recorded the pressure fluctuations at the same locations as in the experiments, that is all the $K 20$ to $K 29$ are located at $0.25 D$ from the cavity center plane that is pointing out-of-the paper and successive sensors (K20 and $K 21, K 21$ and $K 22$ and so on) are equidistant by $0.5 D$ with the first sensor $K 20$ located at $0.25 D$ from the fore-wall of the cavity. It is important to assess the performance of the embedding approach with different metrics, such as now also looking at the SPL generated by the cavity. The SPL at these locations is compared in frames (a) through ( $\mathrm{j}$ ) in figure 16 (solid lines in red). As can be seen from these figures, the present simulations capture the tones similar to those of the experiments. The broadband noise levels also follow closely with those of the experiments. The peaks of these spectra are quasi-invariant as we move along the cavity and their values are compared in table 3 . In this table, values obtained from Rossiter's formula and the reference LES are taken from the article by Larchevêque [14]. The deviation of the frequency values from Rossiter's formula are shown in parenthesis with a negative sign for an under-predicted value and a positive sign for an over-predicted value. As can be seen from this table the first two modes are under-predicted and the other two modes are over-predicted. The over-prediction is somewhat larger in comparison to the reference LES. Next, in order to understand the spatial variation of the tones the BISPL values are computed with a band-width of $100 \mathrm{~Hz}$ and centered around each of the tones, the values of bands of integration are the same as those of the reference [14]. Frames (a) through (d) of figure 17 show the spatial evolution of each of the modes (solid lines in red). The spatial variation of these modes is captured well by the present simulations and compares well with the reference data. Further, all the frequencies in the range of 50 to $2000 \mathrm{~Hz}$ are integrated and the spatial variation is shown in frame (e) of this figure. A good match is obtained with the reference data for the integrated frequencies over this range. Frame (f) of this figure compares the variation of OASPL with the reference data. As can be seen from this figure, the OASPL shows a good match both qualitatively and quantitatively with the reference data with a maximum deviation of $2.6 \mathrm{~dB}$. Further, from the frames (e) and (f) it can be noted that most of the energy is contained in the frequencies over the range 50 to 2000 (containing all the tones).

\subsubsection{Effect of the Incoming Boundary Layer Thickness}

In the experiments it was noted that the leading edge of the rig that houses the cavity caused a recirculation bubble to develop. This had the effect of making the boundary layer thicker. In this section, we present results with an incoming turbulent boundary layer thickness twice that of the previous simulation as well as the reference LES in order to assess the sensitivity of the results to the state of incoming boundary layer. The mesh resolution is kept the same and the solution and statistics collections are the same as in the previous simulation. The normalised streamwise and wall-normal velocity profiles are shown in frames (a) and (b) of 
Table 3 Frequencies $(\mathrm{Hz})$ of pressure spectra peaks

\begin{tabular}{l|l|l|l|l}
\hline Peak number & 1 & 2 & 3 & 4 \\
\hline Rossiter's formula & 148 & 357 & 566 & 775 \\
Experiments & $135(-13)$ & $350(-7)$ & $590(+24)$ & $820(+45)$ \\
Reference LES & $125(-23)$ & $355(-2)$ & $575(+9)$ & $815(+40)$ \\
Present & $117(-31)$ & $353(-4)$ & $624(+58)$ & $860(+85)$ \\
Present, $2 \delta_{99}$ & $117(-31)$ & $353(-4)$ & $586(+20)$ & $819(+44)$ \\
\hline
\end{tabular}

figure 14 with dash dot lines in blue. The thicker incoming boundary layer can be noted from the profile at $x / D=0$. The fore part of the cavity $(x / D<2)$ does not show the effect of the thicker boundary layer. The effect of the thicker boundary layer can be seen at all the locations above the cavity $(y / D>0)$ and in the aft part of the cavity $(x / D>2)$. The wall-normal velocity components show small differences because of a thicker incoming boundary layer especially in the aft part of the cavity. The Reynolds shear stresses and the turbulent kinetic energy profiles are shown in frames (a) and (b) of figure 15 with dash dot lines in blue. Qualitatively the profiles are similar to those of the previous simulation, however, the peak values of the shear are smaller at all the locations in the cavity. This could be explained based on the thickness of the incoming turbulent boundary layer. The shear produced by a thicker boundary layer is smaller when compared to the previous simulation. The turbulent kinetic energy profiles also show qualitatively similar profiles with not much difference in the peak values, rather a deviation in the aft part of the cavity $(x / D>2$ and $y / D<0)$. Next, we turn our attention to understand the effect of thickness of the boundary layer on pressure fluctuations. The SPL are plotted along the cavity floor, similar to the previous case, in frames (a) through (j) in figure 16 with dash dot lines in black. As can be seen from these figures the tones and broadband noise show lower levels when compared with the previous simulation (solid lines in red) except for the third tone. Interestingly, the present SPL seem to be closer to the experimental SPL (solid lines in blue). The better match with experiments for the thicker incoming boundary layer is likely due to the fact that the incoming boundary layer was also thicker in the experiments, owing to a recirculation bubble at the leading-edge of the cavity rig. The frequencies of the tones are extracted and presented in table 3 . The first and second modes did not change when compared with the previous simulation but the third and fourth modes have moved closer to the experimental values with a difference of 20 and $44 \mathrm{~Hz}$ respectively. The lower values of SPL are directly reflected also in the BISPL plots in frames (a) through (f) for each of the modes and in the OASPL shown with dash dot lines in black. The OASPL shows a very good match with the experiments at $x / D \leq 1.5$ and thereafter it shows a small under-prediction. The OASPL, still shows a maximum difference of $2.8 d B$ when compared with the experiments, however, it is an under-prediction in the present case.

\section{Conclusions and Outlook}

A framework has been developed within the OpenFOAM $[47,26]$ solver to embed LES regions inside RANS regions. The DFM is used to generate synthetic turbu- 


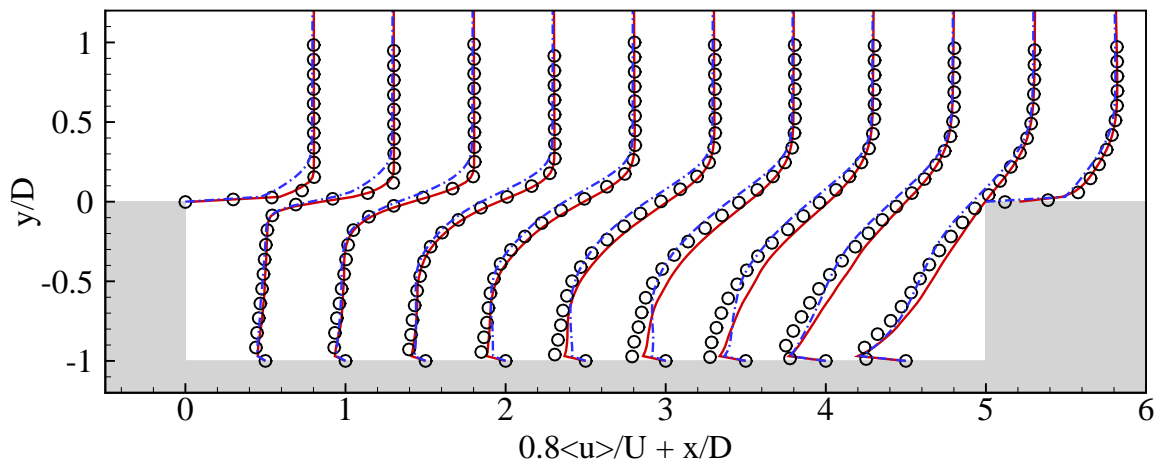

(a) $\langle u\rangle / U$

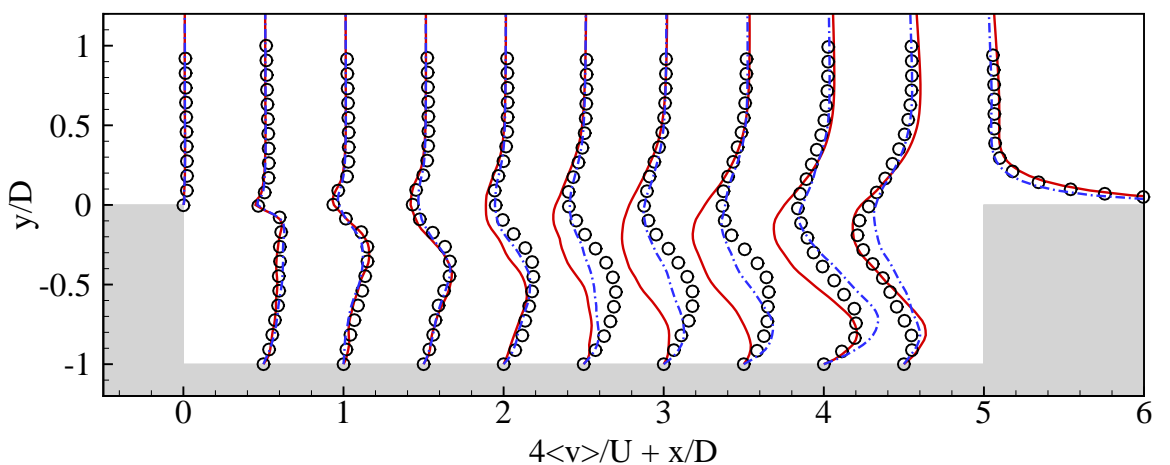

(b) $\langle v\rangle / U$

Fig. 14 Mean velocity profiles on the cavity center plane for reference LES [14] (symbols), embedded LES-RANS (solid lines in red) and embedded LES-RANS with a thicker incoming boundary layer (dash dot lines in blue).

lence at the LES interface with the mean profiles and other inputs obtained from the RANS interface in a fully coupled manner and allowing for arbitrary meshes at the interface. Spatially developing turbulent channel flow at $R e_{\tau}=395$ was used as a development case to validate the framework and mean and second-order statistics were compared with the DNS data, resulting in good agreement. Next, flow over a surface-mounted vertical fence was simulated at $R e=3000$. The importance of introducing turbulence fluctuations using DFM was demonstrated by simulating cases with and without feeding fluctuations at the LES interface and it was observed that the flow relaminarises upstream of the fence and the secondorder statistics do not match with the DNS data at several locations downstream of the fence if no fluctuations were added at the interface. Feeding turbulence fluctuations and placing the LES interface at six fence-heights before the fence gave best agreement with the DNS data at all sampling locations considered. The location of the mean separation region for the primary recirculation bubble behind the fence also matched the DNS data well.

A good match with the experimental data was obtained for the inclined spoiler configuration. This case introduced three-dimensionality because of the finite- 


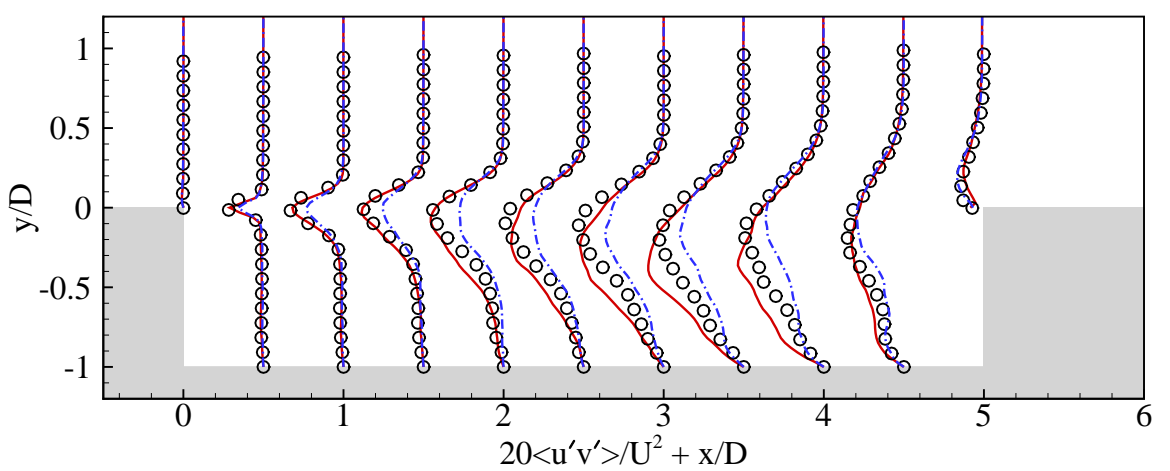

(a) $\left\langle u^{\prime} v^{\prime}\right\rangle / U^{2}$

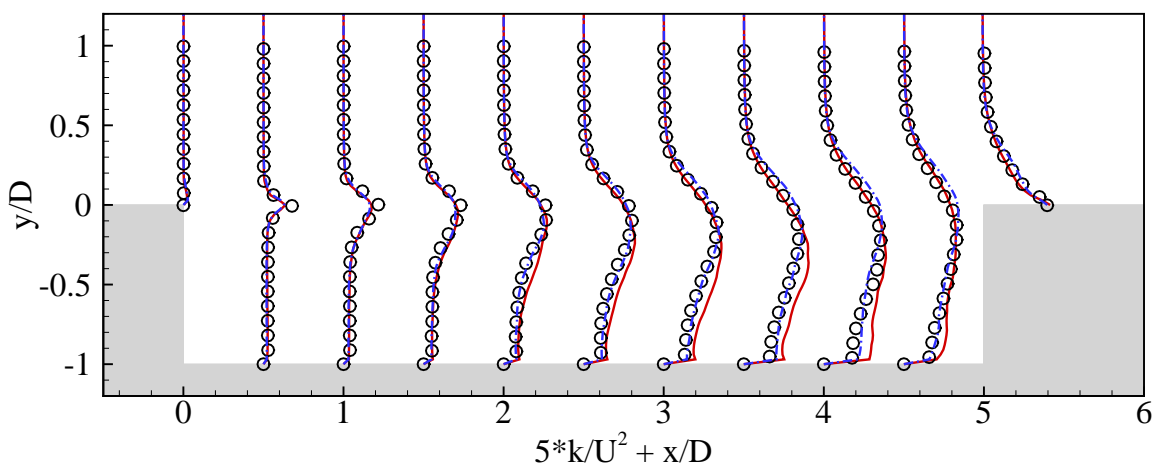

(b) $k / U^{2}$

Fig. 15 Second-order statistics of velocity on the cavity center plane for LES [14] (symbols), embedded LES-RANS (solid lines in red) and embedded LES-RANS with a thicker incoming boundary layer (dash dot lines in blue).

width and a realistic inclination of the spoiler when compared with the previous fence case. As noted in the channel flow simulation, although the Reynolds stress profiles are not perfect when compared with the DNS results, we still get much better predictions in the vertical fence and inclined spoiler simulations when using fluctuations than when they were not used. This indicates that it is most important that there are fluctuations, but may be it is not crucial that they are perfectly presenting the real upstream flow. Finally, the open cavity case showed good agreement with reference data for the mean velocity, Reynolds stresses, and turbulent kinetic energy with the reference data on the cavity center plane. The tones of the pressure spectra were predicted reasonably well and the maximum deviation of the OASPL was found to be only $2.6 \mathrm{~dB}$ when compared with the experiments. A thicker incoming boundary layer was considered in order to account for the effect of the recirculation bubble on the leading edge of the cavity rig. The simulation with a thicker incoming boundary layer captured the third-peak in the spectra quite well in terms of both the frequency and the amplitude. The methodology presented here could be improved in future by considering a more powerful DFM that will reduce the adaptation length required in converting the generated 
synthetic turbulence to a realistic turbulent flow. Further, future simulations could consider testing of the following, (a) using other SGS models in the LES region to identify benefits, for example, in terms of capturing anisotropy of the unresolved scales. (b) simulation of the entire rig geometry for the open cavity case, using the present embedding approach, to accurately capture the incoming boundary layer.

Acknowledgements This work was performed with support from Airbus Group Innovations and BAE Systems through the "Simulations at Off-Design (SimOD) conditions" project. The authors also acknowledge the computational support provided through the IRIDIS4 High Performance Computing Facility at the University of Southampton, and the ARCHER Supercomputer facility at the University of Edinburgh through the UK Turbulence Consortium under EPSRC grant EP/L000261/1. Fruitful discussions with Professor Michael A. Leschziner are gratefully acknowledged. We are grateful to Dr. Lionel Larchevêque for providing us with the open cavity data from his simulations for comparison purposes. We would like to thank the anonymous reviewers for their questions, comments and suggestions that helped to improve the quality of the present paper.

\section{References}

1. Batten, P., Goldberg, U., Chakravarthy, S.: Interfacing statistical turbulence closures with large-eddy simulation. AIAA Journal 42(3), 485-492 (2004)

2. Deck, S.: Recent improvements in the zonal detached eddy simulation (ZDES) formulation. Theoretical and Computational Fluid Dynamics 26(6), 523-550 (2012)

3. Fasel, H.F., Von Terzi, D.A., Sandberg, R.D.: A methodology for simulating compressible turbulent flows. Journal of Applied Mechanics 73(3), 405-412 (2006)

4. Fröhlich, J., von Terzi, D.: Hybrid LES/RANS methods for the simulation of turbulent flows. Progress in Aerospace Sciences 44(5), 349-377 (2008)

5. Gloerfelt, X., Bogey, C., Bailly, C., Juve, D.: Aerodynamic noise induced by laminar and turbulent boundary layers over rectangular cavities. AIAA paper 2476(2002), 1-12 (2002)

6. Grace, S.: An overview of computational aeroacoustic techniques applied to cavity noise prediction. AIAA paper 510(2001), 1-13 (2001)

7. Henshaw, M. J. de C.: M219 cavity case: Verification and validation data for computational unsteady aerodynamics. Tech. rep., RTO-TR-26, AC/323 (AVT) TP/19, QinetiQ, UK (2002)

8. Kim, D.H., Choi, J.H., Kwon, O.J.: Detached eddy simulation of weapons bay flows and store separation. Computers \& Fluids 121, 1-10 (2015)

9. Kim, Y., Castro, I.P., Xie, Z.T.: Divergence-free turbulence inflow conditions for large-eddy simulations with incompressible flow solvers. Computers \& Fluids 84, 56-68 (2013)

10. Klein, M., Sadiki, A., Janicka, J.: A digital filter based generation of inflow data for spatially developing direct numerical or large eddy simulations. Journal of Computational Physics 186(2), 652-665 (2003)

11. Komerath, N.M., Ahuja, K.K., Chambers, F.W.: Prediction and measurement of flows over cavities-A survey. In: 25th AIAA Aerospace Sciences Meeting, vol. 1 (1987)

12. König, D., Meinke, M., Schröder, W.: Embedded LES-to-RANS boundary in zonal simulations. Journal of Turbulence (11) (2010)

13. Krishnamurty, K.: Acoustic radiation from two-dimensional rectangular cutouts in aerodynamic surfaces. Tech. rep., NACA TN 3487 (1955)

14. Larchevêque, L., Sagaut, P., Le, T.H., Comte, P.: Large-eddy simulation of a compressible flow in a three-dimensional open cavity at high Reynolds number. Journal of Fluid Mechanics 516, 265-301 (2004)

15. Larchevêque, L., Sagaut, P., Mary, I., Labbé, O., Comte, P.: Large-eddy simulation of a compressible flow past a deep cavity. Physics of Fluids 15(1), 193-210 (2003)

16. Launder, B.E., Sharma, B.I.: Application of the energy-dissipation model of turbulence to the calculation of flow near a spinning disc. Letters in Heat and Mass Transfer 1(2), 131-137 (1974)

17. Lawson, S.J., Barakos, G.N.: Computational fluid dynamics analyses of flow over weaponsbay geometries. Journal of Aircraft 47(5), 1605-1623 (2010) 
18. Lawson, S.J., Barakos, G.N.: Review of numerical simulations for high-speed, turbulent cavity flows. Progress in Aerospace Sciences 47(3), 186-216 (2011)

19. Lund, T.S., Wu, X., Squires, K.D.: Generation of turbulent inflow data for spatiallydeveloping boundary layer simulations. Journal of Computational Physics 140(2), 233-258 (1998)

20. Mathey, F.: Aerodynamic noise simulation of the flow past an airfoil trailing-edge using a hybrid zonal RANS-LES. Computers \& Fluids 37(7), 836-843 (2008)

21. Medic, G., Kalitzin, G., You, D., Weide, V.D., Alonso, J.J., Pitsch, H.: Integrated RANSLES computation of an entire gas turbine jet engine. In: 45th AIAA Aerospace Sciences Meeting and Exhibit, pp. 2007-1117 (2008)

22. Menter, F.R.: Two-equation eddy-viscosity turbulence models for engineering applications. AIAA Journal 32(8), 1598-1605 (1994)

23. Moser, R.D., Kim, J., Mansour, N.N.: Direct numerical simulation of turbulent channel flow up to $R e=590$. Physics of Fluids 11(4), 943-945 (1999). URL http://turbulence. ices.utexas.edu/MKM_1999.html

24. Nagano, Y., Pei, C.Q., Hattori, H.: A new low-Reynolds-number one-equation model of turbulence. Flow, Turbulence and Combustion 63(1-4), 135-151 (2000)

25. Nedić, J., Ganapathisubramani, B., Vassilicos, J.C., Boree, J., Brizzi, L.E., Spohn, A.: Aeroacoustic performance of fractal spoilers. AIAA Journal 50(12), 2695-2710 (2012)

26. OpenFOAM: OpenCFD Ltd., The open source CFD toolbox. URL http://www . openf oam. com

27. Orellano, A., Wengle, H.: Numerical simulation (DNS and LES) of manipulated turbulent boundary layer flow over a surface-mounted fence. European Journal of MechanicsB/Fluids 19(5), 765-788 (2000)

28. Pope, S.B.: Turbulent flows. Cambridge University Press (2000)

29. Quéméré, P., Sagaut, P.: Zonal multi-domain RANS/LES simulations of turbulent flows. International Journal for Numerical Methods in Fluids 40(7), 903-925 (2002)

30. Richez, F., Mary, I., Gleize, V., Basdevant, C.: Zonal RANS/LES coupling simulation of a transitional and separated flow around an airfoil near stall. Theoretical and Computational Fluid Dynamics 22(3-4), 305-315 (2008)

31. Rockwell, D., Naudascher, E.: Review-self-sustaining oscillations of flow past cavities. Journal of Fluids Engineering 100(2), 152-165 (1978)

32. Roidl, B., Meinke, M., Schröder, W.: A zonal RANS-LES method for compressible flows. Computers \& Fluids 67, 1-15 (2012)

33. Roidl, B., Meinke, M., Schröder, W.: A reformulated synthetic turbulence generation method for a zonal RANS-LES method and its application to zero-pressure gradient boundary layers. International Journal of Heat and Fluid Flow 44, 28-40 (2013)

34. Roidl, B., Meinke, M., Schröder, W.: Boundary layers affected by different pressure gradients investigated computationally by a zonal RANS-LES method. International Journal of Heat and Fluid Flow 45, 1-13 (2014)

35. Roshko, A.: Some measurements of flow in a rectangular cutout. Tech. rep., NACA TN 3488 (1955)

36. Rossiter, J.E.: Wind tunnel experiments on the flow over rectangular cavities at subsonic and transonic speeds. Tech. rep., Ministry of Aviation; Royal Aircraft Establishment; RAE Farnborough (1964)

37. Rowley, C.W., Colonius, T., Basu, A.J.: On self-sustained oscillations in two-dimensional compressible flow over rectangular cavities. Journal of Fluid Mechanics 455, 315-346 (2002)

38. Sagaut, P., Deck, S., Terracol, M.: Multiscale and multiresolution approaches in turbulence. World Scientific (2006)

39. Schlüter, J.U., Wu, X., Kim, S., Alonso, J.J., Pitsch, H.: Coupled RANS-LES computation of a compressor and combustor in a gas turbine engine. In: Proceedings of 40th AIAA/ASME/SAE/ASEE Joint Propulsion Conference and Exhibit, Lauderdale, FL (2004)

40. Schlüter, J.U., Wu, X., Kim, S., Shankaran, S., Alonso, J.J., Pitsch, H.: A framework for coupling Reynolds-averaged with large-eddy simulations for gas turbine applications. Journal of Fluids Engineering 127(4), 806-815 (2005)

41. Smagorinsky, J.: General circulation experiments with the primitive equations: I. the basic experiment. Monthly Weather Review 91(3), 99-164 (1963)

42. Spalart, P.R.: Detached-eddy simulation. Annual Review of Fluid Mechanics 41, 181-202 (2009) 
43. Spalart, P.R., Deck, S., Shur, M.L., Squires, K.D., Strelets, M.K., Travin, A.: A new version of detached-eddy simulation, resistant to ambiguous grid densities. Theoretical and Computational Fluid Dynamics 20(3), 181-195 (2006)

44. Touber, E., Sandham, N.D.: Large-eddy simulation of low-frequency unsteadiness in a turbulent shock-induced separation bubble. Theoretical and Computational Fluid Dynamics 23(2), 79-107 (2009)

45. Van Driest, E.R.: On turbulent flow near a wall. Journal of the Aeronautical Sciences (Institute of the Aeronautical Sciences) 23(11) (2012)

46. Weinmann, M., Sandberg, R.D., Doolan, C.: Tandem cylinder flow and noise predictions using a hybrid RANS/LES approach. International Journal of Heat and Fluid Flow 50, 263-278 (2014)

47. Weller, H.G., Tabor, G., Jasak, H., Fureby, C.: A tensorial approach to computational continuum mechanics using object-oriented techniques. Computers in Physics 12(6), 620$631(1998)$

48. Xiao, H., Jenny, P.: A consistent dual-mesh framework for hybrid LES/RANS modeling. Journal of Computational Physics 231(4), 1848-1865 (2012)

49. Xie, Z.T., Castro, I.P.: Efficient generation of inflow conditions for large eddy simulation of street-scale flows. Flow, Turbulence and Combustion 81(3), 449-470 (2008)

50. Zhang, Q., Schröder, W., Meinke, M.: A zonal RANS-LES method to determine the flow over a high-lift configuration. Computers \& Fluids 39(7), 1241-1253 (2010) 


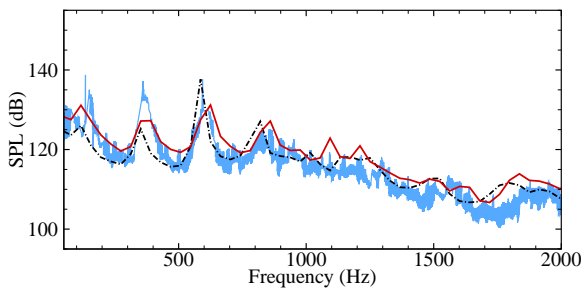

(a) K20

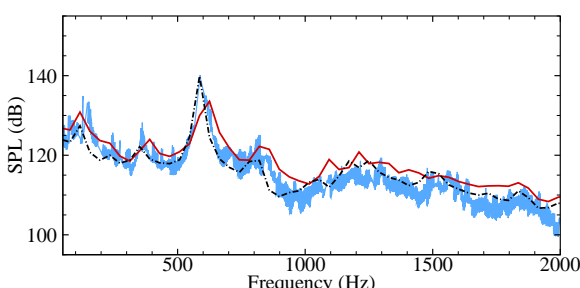

(c) K22

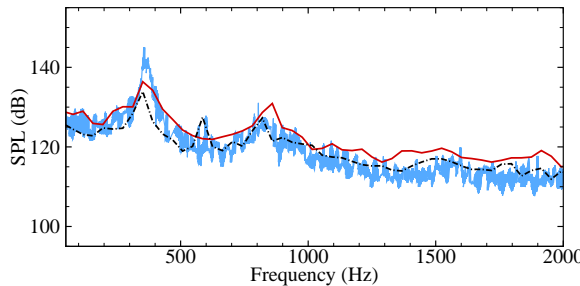

(e) K24

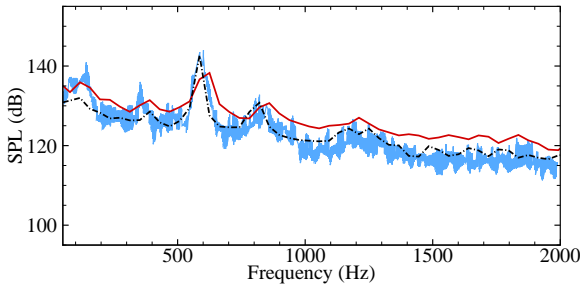

(g) K26

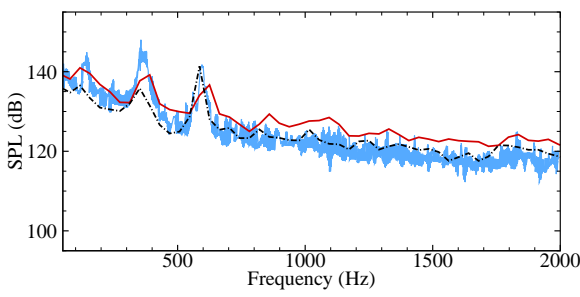

(i) $\mathrm{K} 28$

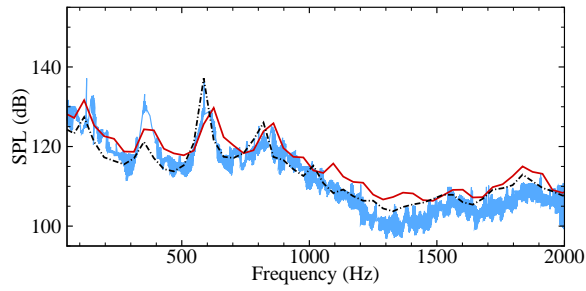

(b) K21

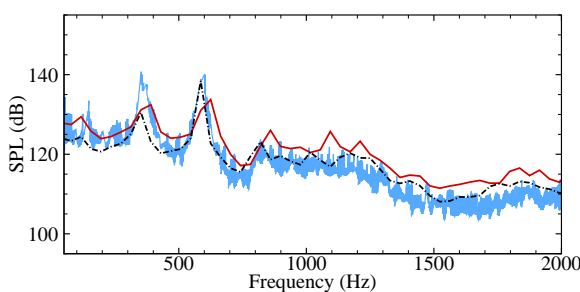

(d) K23

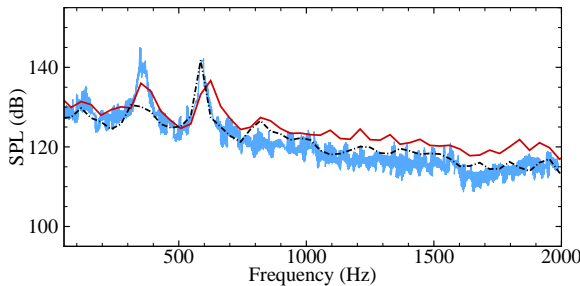

(f) $\mathrm{K} 25$

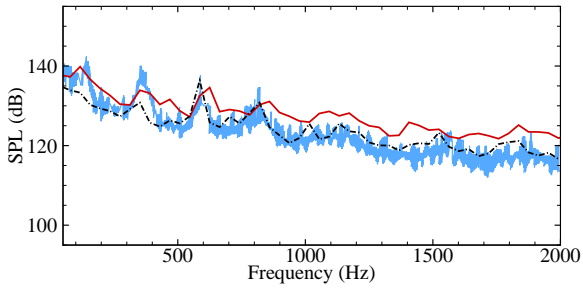

(h) K27

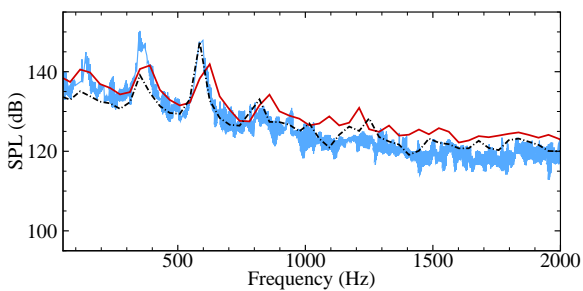

(j) K29

Fig. 16 Comparison of SPL spectra for the present simulations (solid lines in red), present simulations with a thicker incoming boundary layer (dash dot lines in black) and for the experiment [7] (blue lines) for several pressure probes. 


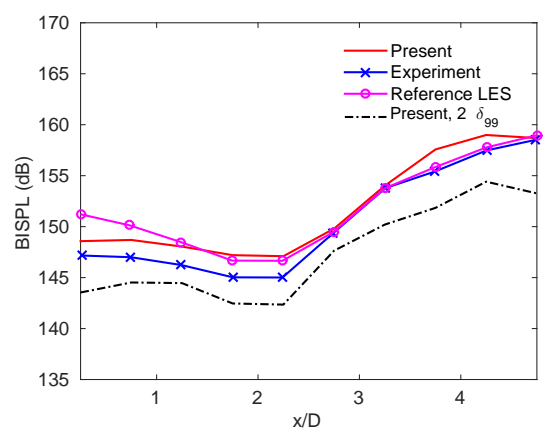

(a) Mode 1

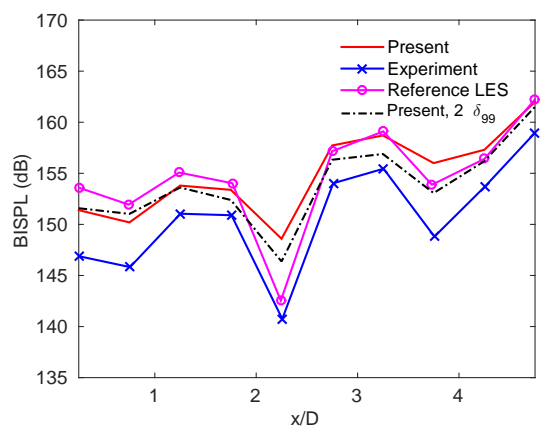

(c) Mode 3

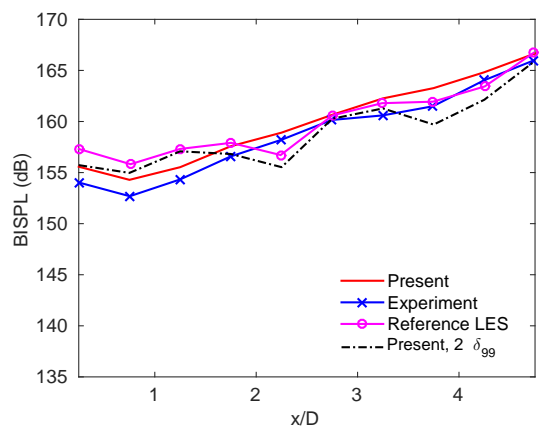

(e) Integrated over 50 to $2000 \mathrm{~Hz}$

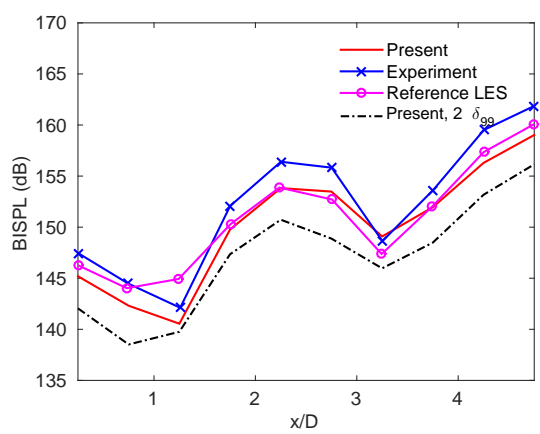

(b) Mode 2

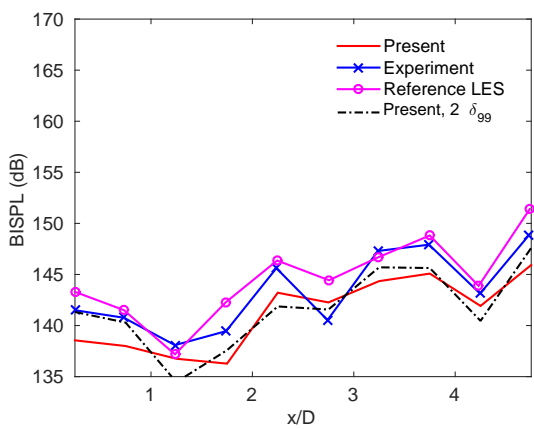

(d) Mode 4

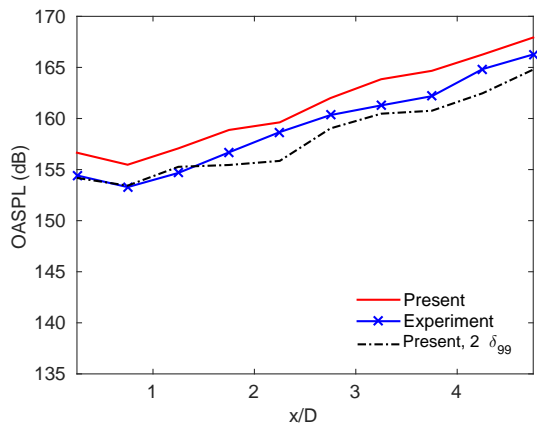

(f) OASPL

Fig. 17 Comparison of band-integrated and overall sound pressure levels for the present simulations (solid lines in red), present simulations with a thicker incoming turbulent boundary layer (dash dot lines in black), reference LES [14] (magenta lines with o symbols) and the experiment [7] (blue lines with x symbols). 


\section{University Library}

\section{- M M I N E R VA A gateway to Melbourne's research publications}

Minerva Access is the Institutional Repository of The University of Melbourne

Author/s:

Anupindi, K;Sandberg, RD

Title:

Implementation and Evaluation of an Embedded LES-RANS Solver

Date:

2017-04-01

Citation:

Anupindi, K. \& Sandberg, R. D. (2017). Implementation and Evaluation of an Embedded LES-RANS Solver. FLOW TURBULENCE AND COMBUSTION, 98 (3), pp.697-724. https:// doi.org/10.1007/s10494-016-9787-5.

Persistent Link:

http://hdl.handle.net/11343/282978 